\title{
KOLMOGOROV BOUNDS FOR THE NORMAL APPROXIMATION OF THE NUMBER OF TRIANGLES IN THE ERDŐS-RÉNYI RANDOM GRAPH
}

\author{
AdRIAN RÖLLIN \\ National University of Singapore
}

\begin{abstract}
We bound the error for the normal approximation of the number of triangles in the Erdős-Rényi random graph with respect to the Kolmogorov metric. Our bounds match the best available Wasserstein-bounds obtained by Barbour. Karoński and Ruciński (1989), resolving a long-standing open problem. The proofs are based on a new variant of the Stein-Tikhomirov method - a combination of Stein's method and characteristic functions introduced by Tikhomirov (1980).
\end{abstract}

\section{INTRODUCTION}

Consider the Erdős-Rényi random graph $G(n, p)$, where $p$ is allowed to depend on $n$. The conditions under which subgraph counts exhibit asymptotic normality were fully characterised by Ruciński (1988); in the particular case of triangles, a normal limit holds if and only if

$$
\lim _{n \rightarrow \infty} n p=\infty \quad \text { and } \quad \lim _{n \rightarrow \infty} n^{2}(1-p)=\infty .
$$

This result was complemented by corresponding rates of convergence bv Barbour, Karoński and Ruciński (1989) for the Wasserstein metric $d_{\mathrm{W}}$. Let $T$ denote the number of triangles in $G(n, p)$, and let $W=(T-\mathbb{E} T) / \sqrt{\operatorname{Var} T}$ denotes the centred and normalised number of triangles; Barbour. Karoński and Ruciński (1989) showed that there is a universal constant $C$ such that

$$
d_{\mathrm{W}}(\mathscr{L}(W), \Phi) \leqslant \begin{cases}\frac{C}{n(1-p)^{1 / 2}} & \text { if } 1 / 2<p<1, \\ \frac{C}{n p^{1 / 2}} & \text { if } n^{-1 / 2}<p \leqslant 1 / 2, \\ \frac{C}{n^{3 / 2} p^{3 / 2}} & \text { if } 0<p \leqslant n^{-1 / 2},\end{cases}
$$

where $\Phi$ is the standard normal distribution function. This bound indeed goes to zero exactly under the conditions of asymptotic normality obtained by Ruciński (1988).

Obtaining bounds with respect to the Kolmogorov metric $d_{K}$ has turned out to be much harder, with no progress until recently. A straightforward bound, as is well known, can be obtained through the relation

$$
d_{\mathrm{K}}(\mathscr{L}(W), \Phi) \leqslant \sqrt{d_{\mathrm{W}}(\mathscr{L}(W), \Phi)}
$$

(see e.g. Ross (2011, Proposition 1.2)). To the best of our knowledge, the first result that improves on (1.3) was obtained by Röllin and Ross (2015, Theorem 4.11), which is a stronger total variation approximation by a translated Poisson distribution. Better rates for the Kolmogorov metric were obtained later by Krokowski, Reichenbachs and Thäle (2017) using Stein's method in combination with Malliavin-type methods.

The following is our main result. 
Theorem 1.1. Let $W$ be the centred and normalised number of triangles in $G(n, p)$. There is a universal constant $C$ such that, for every $n \geqslant 3$ and every $0<p<1$,

$$
d_{\mathrm{K}}(\mathscr{L}(W), \Phi) \leqslant \begin{cases}\frac{C}{n(1-p)^{1 / 2}} & \text { if } 1 / 2<p<1, \\ \frac{C}{n p^{1 / 2}} & \text { if } n^{-1 / 2}<p \leqslant 1 / 2, \\ \frac{C}{n^{3 / 2} p^{3 / 2}} & \text { if } 0<p \leqslant n^{-1 / 2} .\end{cases}
$$

Remark 1.2. In order to analyse the optimality of the bound (1.4), consider the variance of the number of triangles (see Lemma 3.6), which satisfies

$$
\operatorname{Var} T \asymp \begin{cases}n^{4}(1-p) & \text { if } 1 / 2<p<1, \\ n^{4} p^{5} & \text { if } n^{-1 / 2}<p \leqslant 1 / 2, \\ n^{3} p^{3} & \text { if } 0<p \leqslant n^{-1 / 2},\end{cases}
$$

where we write $f(n) \asymp g(n)$ if the ratio $f(n) / g(n)$ is bounded away from 0 and infinity as $n \rightarrow \infty$. If $0<p \leqslant n^{-1 / 2}$, the bound (1.4) is of order $1 / \sqrt{\operatorname{Var} T}$, which is best possible for an integer-valued random variable normalised by its standard deviation. In the other two cases, the dependence between the triangle indicators becomes so strong that the covariance terms change the order of the variance, but the following example shows that the rate in (1.4) is still within what can be expected for sums of random variables with a similar covariance structure.

For $1 \leqslant i<j<k \leqslant n$, let $I_{i j} \sim \operatorname{Be}(p)$ and $I_{i j k} \sim \operatorname{Be}\left(p^{2}\right)$ be independent random variables, and let $X_{i j k}=I_{i j} I_{i j k}$. With $Y=\sum_{1<i<j<k \leqslant n} X_{i j k}$ we have $\mathbb{E} Y=\left(\begin{array}{l}n \\ 3\end{array}\right) p^{3}=\mathbb{E} T$ and

$$
s^{2}:=\operatorname{Var} Y=\left(\begin{array}{l}
n \\
3
\end{array}\right)\left(p^{3}(1-p)^{3}+(n-3) p^{5}(1-p)\right) \asymp \operatorname{Var} T .
$$

Since $Y$ is the sum of $\left(\begin{array}{l}n \\ 2\end{array}\right)$ independent and identically distributed random variables, each being distributed like $I_{12} \sum_{3 \leqslant k \leqslant n} I_{12 k}$, we can apply the Berry-Esseen theorem and obtain

$$
d_{\mathrm{K}}((Y-\mathbb{E} Y) / s, \Phi) \leqslant \frac{C n^{2} \gamma}{s^{3}}, \quad \text { where } \quad \gamma=\mathbb{E}\left|I_{12} \sum_{3 \leqslant k \leqslant n} I_{12 k}-(n-2) p^{3}\right|^{3} .
$$

Now,

$$
\gamma=p \mathbb{E}\left|\sum_{3 \leqslant k \leqslant n}\left(I_{12 k}-p^{2}\right)+(n-2) p^{2}(1-p)\right|^{3}+(1-p)(n-2)^{3} p^{9} .
$$

First, consider the case where $n^{-1 / 2}<p \leqslant 1 / 2$; we can use Bernstein's inequality to conclude that $\sum_{3 \leqslant k \leqslant n}\left(I_{12 k}-p^{2}\right)$ is strongly concentrated around 0 , so that $\gamma \asymp n^{3} p^{7}$. Recalling that $s^{2} \asymp n^{4} p^{5}$, it follows that the bound in (1.5) is of order $n^{-1} p^{-1 / 2}$, which is the same as that of (1.4). In the case where $1 / 2<p<1$, we can bound

$$
\begin{aligned}
& \mathbb{E}\left|\sum_{3 \leqslant k \leqslant n}\left(I_{12 k}-p^{2}\right)+(n-2) p^{2}(1-p)\right|^{3} \\
& \quad \leqslant \mathbb{E}\left|\sum_{3 \leqslant k \leqslant n}\left(I_{12 k}-p^{2}\right)\right|^{3}+(n-2)^{3} p^{6}(1-p)^{3} \\
& \quad \leqslant C\left(\max \left\{n(1-p), n^{2}(1-p)^{2}\right\}\right)^{3 / 4}+C n^{3}(1-p)^{3} .
\end{aligned}
$$


If $\lim n(1-p)=\infty$, the bound (1.6) is of order $n^{3}(1-p)^{3}$, and so $\gamma \asymp n^{3}(1-p)$. If $\lim \sup n(1-p)<\infty$, we also have $\gamma \asymp n^{3}(1-p)$, as $\lim n^{2}(1-p)=\infty$ by (1.1). Recalling that $s^{2} \asymp n^{4}(1-p)$, we conclude that the bound in (1.5) is of order $n^{-1}(1-p)^{-1 / 2}$, which, again, is the same as that of (1.4).

Since the Berry-Esseen theorem gives optimal rates in general, this example strongly indicates that (1.4) indeed yields the correct rate of convergence also for $n^{-1 / 2}<p \leqslant 1 / 2$ and $1 / 2<p<1$.

\section{THE STEIN-TIKHOMIROV METHOD}

Our proof is based on a method introduced by Tikhomirov (1980), who takes elements from Stein's method, initiated by Stein (1972), and combines them with characteristic functions. As far as we can tell, the Stein-Tikhomirov method has only been successfully applied to prove CLTs for sums of random variables with temporal or spatial dependence and some mixing conditions; see for example Bulinskii (1996), who obtained CLTs for associated random variables index by the $d$-dimensional lattice assuming an exponential decay of the covariances. In order to apply the Stein-Tikhomirov method to triangle counts, we develop in this section a new abstract theorem by combining Tikhomirov's approach with ideas from the more recent literature around Stein's method; we use, in particular, Stein couplings from Chen and Röllin (2010).

To this end, we say a triple of random variables $\left(W, W^{\prime}, G\right)$ is a Stein coupling if

$$
\mathbb{E}\left\{G f\left(W^{\prime}\right)-G f(W)\right\}=\mathbb{E}\{W f(W)\}
$$

for all functions $f$ for which the expectations exist. We refer to Chen and Röllin (2010) for examples and applications of Stein couplings.

Although the random variables of interest are real valued, we will need to consider complex valued random variables due to the use of characteristic functions. If $z=x+i y \in$ $\mathbb{C}$, we denote by $z^{*}=x-i y$ its complex conjugate. If $X=X_{1}+i X_{2}$ is a complex random variables, we define as usual

$$
\operatorname{Var} X=\mathbb{E}\left\{(X-\mathbb{E} X)(X-\mathbb{E} X)^{*}\right\},
$$

and if $Y$ is another complex random variable, we define

$$
\operatorname{Cov}(X, Y)=\mathbb{E}\left\{(X-\mathbb{E} X)(Y-\mathbb{E} Y)^{*}\right\}
$$

All the usual properties of the variance and covariance functions remain the same, except that $\operatorname{Cov}(X, Y)=\operatorname{Cov}(Y, X)^{*}$, from which we conclude that $\operatorname{Var}(a X)=|a|^{2} \operatorname{Var}(X)$ and $\operatorname{Cov}(a X, b Y)=a b^{*} \operatorname{Cov}(X, Y)$ for any $a, b \in \mathbb{C}$.

The following is our main result in this section.

Theorem 2.1. Let $\left(W, W^{\prime}, G\right)$ be a Stein coupling with $\operatorname{Var} W=1$. Then

$$
\sup _{x \in \mathbb{R}}|\mathbb{P}[W \leqslant x]-\mathbb{P}[Z \leqslant x]| \leqslant 0.38 r_{1}+3.05 \tilde{r}_{1}+0.64 r_{2}\left(1+2 \log _{+} \frac{1}{2 \tilde{r}_{1}}\right)
$$

where, with $D=W^{\prime}-W$,

$$
r_{1}=\mathbb{E}\left|G D^{2}\right|, \quad r_{2}=\sup _{t \neq 0} \frac{\left[\operatorname{Var} \mathbb{E}^{W}\left(G\left(e^{i t D}-1\right)\right)\right]^{1 / 2}}{|t|},
$$


and where $\tilde{r}_{1} \geqslant r_{1}$ is arbitrary. If, in addition, there are random variables $\tilde{D}, S$ and $W^{\prime \prime}$ such that $\mathbb{E}^{W}(G \tilde{D})=\mathbb{E}^{W}(G D)$, such that $\mathbb{E}^{W} S=1$, and such that $\mathbb{E}^{W^{\prime \prime}}(G \tilde{D})=\mathbb{E}^{W^{\prime \prime}} S$, then

$$
\sup _{x \in \mathbb{R}}|\mathbb{P}[W \leqslant x]-\mathbb{P}[Z \leqslant x]| \leqslant 0.76 r_{3}+6.10 \tilde{r}_{3}+0.64 \frac{r_{4}}{\tilde{r}_{3}},
$$

where, with $D^{\prime}=W^{\prime \prime}-W$,

$$
\begin{aligned}
r_{3}= & \frac{1}{2} \mathbb{E}\left|G D^{2}\right|+\mathbb{E}\left|G \tilde{D} D^{\prime}\right|+\mathbb{E}\left|S D^{\prime}\right|, \\
r_{4}= & \sup _{t \neq 0} \frac{\left[\operatorname{Var} \mathbb{E}^{W}\left(G\left(e^{i t D}-1-i t D\right)\right)\right]^{1 / 2}}{t^{2}}+\sup _{t \neq 0} \frac{\left[\operatorname{Var} \mathbb{E}^{W}\left(G \tilde{D}\left(e^{i t D^{\prime}}-1\right)\right)\right]^{1 / 2}}{|t|} \\
& +\sup _{t \neq 0} \frac{\left[\operatorname{Var} \mathbb{E}^{W}\left(S\left(e^{i t D^{\prime}}-1\right)\right)\right]^{1 / 2}}{|t|},
\end{aligned}
$$

and where $\tilde{r}_{3} \geqslant r_{3}$ is arbitrary.

Although the terms $r_{1}$ and $r_{2}$ in (2.2) are much simpler than the terms $r_{3}$ and $r_{4}$ in (2.3), the bound (2.2) comes at the cost of an additional logarithmic term, so that in order to prove Theorem 1.1, we will have to make use of (2.3).

To prove Theorem 2.1, we will use the following, classic smoothing lemma due to Esseen; see, for example, Ibragimov and Linnik (1971, Theorem 1.5.2, p. 27/28).

Lemma 2.2. Let $W$ be a random variable with characteristic function $\varphi(t)=\mathbb{E} e^{i t W}$, and let $Z$ have a standard normal distribution. Then, for any $T>0$,

$$
\sup _{x \in \mathbb{R}}|\mathbb{P}[W \leqslant x]-\mathbb{P}[Z \leqslant x]| \leqslant \frac{1}{\pi} \int_{-T}^{T} \frac{\left|\varphi(t)-e^{-t^{2} / 2}\right|}{|t|} d t+\frac{24}{\pi \sqrt{2 \pi} T} .
$$

The next lemma is an adapted and more explicit version of what is used implicitly by Tikhomirov (1980) (see in particular Equation (3.22) therein)

Lemma 2.3. Let $W$ be an integrable random variable, and assume its characteristic function $\varphi(t)=\mathbb{E} e^{i t W}$ satisfies the differential equation

$$
\varphi^{\prime}(t)=-t(1+a(t)) \varphi(t)+b(t), \quad t \in \mathbb{R},
$$

where $a(t)$ and $b(t)$ are (possibly complex valued) functions satisfying

$$
|a(t)| \leqslant A_{0}+A_{1}|t|, \quad|b(t)| \leqslant B_{0}+B_{1}|t|+B_{2} t^{2}
$$

for some non-negative constants $A_{0}<1 / 2, A_{1}, B_{0}, B_{1}$ and $B_{2}$. Then, for any $t \geqslant$ $2 A_{1} /\left(1-2 A_{0}\right)$,

$$
\begin{aligned}
\sup _{x \in \mathbb{R}} \mid \mathbb{P} & {[W \leqslant x]-\mathbb{P}[Z \leqslant x] \mid } \\
& \leqslant \frac{2}{\pi} A_{0}+\frac{4}{3 \sqrt{\pi}} A_{1}+\frac{\sqrt{\pi}}{2} B_{0}+\frac{2}{\pi} B_{1}\left(1+2 \log _{+} \frac{1}{2 t}\right)+\frac{4}{\pi} \frac{B_{2}}{t}+\frac{24 t}{\pi \sqrt{2 \pi}} .
\end{aligned}
$$

Proof. The ODE

$$
\varphi^{\prime}(t)=\tilde{a} \varphi(t)+b(t), \quad t \in \mathbb{R},
$$

with boundary condition $\varphi(0)=1$ has solution

$$
\varphi(t)=\exp \left(\int_{0}^{t} \tilde{a}(u) d u\right)+\int_{0}^{t} \exp \left(\int_{u}^{t} \tilde{a}(v) d v\right) b(u) d u .
$$


Hence, for $\tilde{a}(t)=-t-t a(t)$ and with

$$
\Delta(t)=\int_{0}^{t} u a(u) d u
$$

we have

$$
\begin{aligned}
\varphi(t) & =\exp \left(-\frac{t^{2}}{2}-\Delta(t)\right)+\exp \left(-\frac{t^{2}}{2}-\Delta(t)\right) \int_{0}^{t} \exp \left(\frac{u^{2}}{2}+\Delta(u)\right) b(u) d u \\
& =\exp \left(-\frac{t^{2}}{2}\right)+R_{1}(t)+R_{2}(t)
\end{aligned}
$$

where

$$
\begin{aligned}
& R_{1}(t)=\exp \left(-\frac{t^{2}}{2}-\Delta(t)\right)-\exp \left(-\frac{t^{2}}{2}\right) \\
& R_{2}(t)=\exp \left(-\frac{t^{2}}{2}-\Delta(t)\right) \int_{0}^{t} \exp \left(\frac{u^{2}}{2}+\Delta(u)\right) b(u) d u
\end{aligned}
$$

Using

$$
e^{x+h}-e^{x}=h \int_{0}^{1} e^{x+h s} d s
$$

we write

$$
R_{1}(t)=-\Delta(t) \int_{0}^{1} \exp \left(-\frac{t^{2}}{2}-s \Delta(t)\right) d s .
$$

Using the bound on $a(t)$,

$$
|\Delta(t)| \leqslant\left|\int_{0}^{t} u a(u) d u\right| \leqslant \frac{A_{0}}{2} t^{2}+\frac{A_{1}}{3}|t|^{3} .
$$

Since

$$
\frac{A_{0}}{2}+\frac{A_{1}}{3}|t| \leqslant \frac{1}{4} \quad \Longleftrightarrow \quad|t| \leqslant \frac{3\left(1-2 A_{0}\right)}{4 A_{1}}=: T_{1},
$$

we have

$$
-\frac{t^{2}}{2}-s \Delta(t) \leqslant-\frac{t^{2}}{4} \quad \text { for }|t| \leqslant T_{1}
$$

for all $0 \leqslant s \leqslant 1$. This yields

$$
\left|R_{1}(t)\right|=\left|\Delta(t) \int_{0}^{1} \exp \left(-\frac{t^{2}}{2}-s \Delta(t)\right) d s\right| \leqslant\left(\frac{A_{0}}{2} t^{2}+\frac{A_{1}}{3}|t|^{3}\right) \exp \left(-\frac{t^{2}}{4}\right) \quad \text { for }|t| \leqslant T_{1} .
$$

In order to bound $R_{2}(t)$, write

$$
R_{2}(t)=\int_{0}^{t} \exp \left(-\left(\frac{t^{2}}{2}-\frac{u^{2}}{2}\right)-(\Delta(t)-\Delta(u))\right) b(u) d u .
$$

Whenever $0 \leqslant u \leqslant t$ or $t \leqslant u \leqslant 0$, we have

$$
|\Delta(t)-\Delta(u)|=\left|\int_{u}^{t} v a(v) d v\right| \leqslant\left(A_{0}+A_{1}|t|\right)\left|\int_{u}^{t} v d v\right| \leqslant \frac{A_{0}+A_{1}|t|}{2}\left(t^{2}-u^{2}\right) .
$$

Thus, since

$$
\frac{A_{0}+A_{1}|t|}{2} \leqslant \frac{1}{4} \Longleftrightarrow|t| \leqslant \frac{1-2 A_{0}}{2 A_{1}}=: T_{2}
$$


we obtain

$$
\left|R_{2}(t)\right| \leqslant \exp \left(-\frac{t^{2}}{4}\right)\left|\int_{0}^{t}\right| b(u)\left|\exp \left(\frac{u^{2}}{4}\right) d u\right| \quad \text { for }|t| \leqslant T_{2} .
$$

Applying the bound on $|b(t)|$, this yields

$$
\begin{aligned}
\left|R_{2}(t)\right| \leqslant & B_{0} \exp \left(-\frac{t^{2}}{4}\right) \int_{0}^{|t|} \exp \left(\frac{u^{2}}{4}\right) d u+B_{1} \exp \left(-\frac{t^{2}}{4}\right) \int_{0}^{|t|} u \exp \left(\frac{u^{2}}{4}\right) d u \\
& \quad+B_{2} \exp \left(-\frac{t^{2}}{4}\right) \int_{0}^{|t|} u^{2} \exp \left(\frac{u^{2}}{4}\right) d u \\
= & B_{0}|F(t / 2)|+2 B_{1}\left(1-e^{-t^{2} / 4}\right)+2 B_{2}|t|\left(1-e^{-t^{2} / 4}\right) \\
\leqslant & B_{0}|F(t / 2)|+2\left(B_{1}+B_{2}|t|\right) \min \left\{1, t^{2} / 4\right\}
\end{aligned}
$$

where $F(t)=e^{-t^{2}} \int_{0}^{t} e^{u^{2}} d u$ is Dawson's function. Now, Lemma 2.2 states that

$$
\sup _{x \in \mathbb{R}}|\mathbb{P}[W \leqslant x]-\mathbb{P}[Z \leqslant x]| \leqslant \frac{1}{\pi} \int_{-T}^{T} \frac{\left|\varphi(t)-e^{-t^{2} / 2}\right|}{|t|} d t+\frac{24}{\pi \sqrt{2 \pi} T} .
$$

Since $\left|\varphi(t)-e^{-t^{2} / 2}\right| \leqslant\left|R_{1}(t)\right|+\left|R_{2}(t)\right|$, we obtain

$$
\begin{aligned}
& \int_{-T}^{T} \frac{\left|\varphi(t)-e^{t^{2} / 2}\right|}{|t|} d t \\
& \leqslant \int_{-T}^{T}\left[\left(\frac{A_{0}}{2}|t|+\frac{A_{1}}{3} t^{2}\right) \exp \left(-\frac{t^{2}}{4}\right)+B_{0} \frac{|F(t / 2)|}{|t|}\right. \\
& \left.+2 B_{1} \frac{\min \left\{1, t^{2} / 4\right\}}{|t|}+2 B_{2} \min \left\{1, t^{2} / 4\right\}\right] d t \\
& \leqslant \int_{-\infty}^{\infty}\left[\left(\frac{A_{0}}{2}|t|+\frac{A_{1}}{3} t^{2}\right) \exp \left(-\frac{t^{2}}{4}\right)+B_{0} \frac{|F(t / 2)|}{|t|}\right] d t \\
& +\int_{-T}^{T}\left[2 B_{1} \frac{\min \left\{1, t^{2} / 4\right\}}{|t|}+2 B_{2} \min \left\{1, t^{2} / 4\right\}\right] d t \\
& \leqslant 2 A_{0}+\frac{4 \sqrt{\pi}}{3} A_{1}+\frac{\pi^{3 / 2}}{2} B_{0}+2 B_{1}\left(1+2 \log _{+}(T / 2)\right)+4 B_{2} T,
\end{aligned}
$$

as long as $T \leqslant T_{1} \wedge T_{2}=T_{2}$. Hence, for any $T \leqslant T_{2}=\left(1-2 A_{0}\right) /\left(2 A_{1}\right)$,

$$
\begin{aligned}
\sup _{x \in \mathbb{R}}|\mathbb{P}[W \leqslant x]-\mathbb{P}[Z \leqslant x]| \\
\quad \leqslant \frac{2}{\pi} A_{0}+\frac{4}{3 \sqrt{\pi}} A_{1}+\frac{\sqrt{\pi}}{2} B_{0}+\frac{2}{\pi} B_{1}\left(1+2 \log _{+}(T / 2)\right)+\frac{4}{\pi} B_{2} T+\frac{24}{\pi T \sqrt{2 \pi}},
\end{aligned}
$$

which, after replacing $T$ by $1 / t$, proves the claim.

Proof of Theorem 2.1. Applying (2.1) to $f(x)=e^{i t x}$ we have

$$
\begin{aligned}
\varphi^{\prime}(t) & =i \mathbb{E}\{W f(W)\}=i \mathbb{E}\left\{G\left(e^{i t D}-1\right) e^{i t W}\right\} \\
& =i \mathbb{E}\left\{G\left(e^{i t D}-1\right)\right\} \varphi(t)+i \mathbb{E}\left\{\left(G\left(e^{i t D}-1\right)-\mathbb{E}\left\{G\left(e^{i t D}-1\right)\right\}\right) e^{i t W}\right\} \\
& =-t(1+a(t)) \varphi(t)+b(t)
\end{aligned}
$$

with

$$
a(t)=\frac{\mathbb{E}\left\{G\left(e^{i t D}-1\right)\right\}}{i t}-1, \quad b(t)=i \mathbb{E}\left\{\left(G\left(e^{i t D}-1\right)-\mathbb{E}\left\{G\left(e^{i t D}-1\right)\right\}\right) e^{i t W}\right\} .
$$


Since $\mathbb{E}(G D)=1$, we have

$$
a(t)=\frac{\mathbb{E}\left\{G\left(e^{i t D}-1-i t D\right)\right\}}{i t},
$$

and thus,

$$
|a(t)| \leqslant \frac{t}{2} \mathbb{E}\left|G D^{2}\right|=0.5 r_{1}|t|=: A_{1}|t|
$$

Moreover,

$$
\begin{aligned}
|b(t)| & \leqslant \mathbb{E}\left|\left(\mathbb{E}^{W}\left\{G\left(e^{i t D}-1\right)\right\}-\mathbb{E}\left\{G\left(e^{i t D}-1\right)\right\}\right)\right| \\
& \leqslant \sqrt{\operatorname{Var} \mathbb{E}^{W}\left\{G\left(e^{i t D}-1\right)\right\}} \leqslant r_{2}|t|=: B_{1}|t| .
\end{aligned}
$$

The first claim now follows from Lemma 2.3 after some straightforward simplifications.

In order to obtain the second claim, add and subtract $t \mathbb{E}\left\{(G D-1) e^{i t W}\right\}$ in (2.5), so that

$$
\begin{aligned}
\varphi^{\prime}(t)= & i \mathbb{E}\left\{G\left(e^{i t D}-1\right)\right\} \varphi(t)-t \mathbb{E}\left\{(G D-1) e^{i t W}\right\} \\
& +i \mathbb{E}\left\{\left(G\left(e^{i t D}-1-i t D\right)-\mathbb{E}\left\{G\left(e^{i t D}-1-i t D\right)\right\}\right) e^{i t W}\right\} .
\end{aligned}
$$

Using the conditions posed on $\tilde{D}, S$ and $W^{\prime \prime}$,

$$
\begin{aligned}
\mathbb{E}\left\{(G D-1) e^{i t W}\right\} & =\mathbb{E}\left\{(G \tilde{D}-S) e^{i t W}\right\} \\
& =\mathbb{E}\left\{(G \tilde{D}-S)\left(e^{i t W}-e^{i t W^{\prime \prime}}\right)\right\} \\
& =\mathbb{E}\left\{(G \tilde{D}-S)\left(1-e^{i t D^{\prime}}\right) e^{i t W}\right\},
\end{aligned}
$$

so that now $\varphi^{\prime}(t)=-t(1+a(t)) \varphi(t)+b(t)$ holds with

$$
\begin{aligned}
& a(t)= \frac{\mathbb{E}\left\{G\left(e^{i t D}-1-i t D\right)\right\}}{i t}-\mathbb{E}\left\{G \tilde{D}\left(e^{i t D^{\prime}}-1\right)\right\}+\mathbb{E}\left\{S\left(e^{i t D^{\prime}}-1\right)\right\} \\
& b(t)=i \mathbb{E}\left\{\left(G\left(e^{i t D}-1-i t D\right)-\mathbb{E}\left\{G\left(e^{i t D}-1-i t D\right)\right\}\right) e^{i t W}\right\} \\
& \quad+t \mathbb{E}\left\{\left(G \tilde{D}\left(e^{i t D^{\prime}}-1\right)-\mathbb{E}\left\{G \tilde{D}\left(e^{i t D^{\prime}}-1\right)\right\}\right) e^{i t W}\right\} \\
&-t \mathbb{E}\left\{\left(S\left(e^{i t D^{\prime}}-1\right)-\mathbb{E}\left\{S\left(e^{i t D^{\prime}}-1\right)\right\}\right) e^{i t W}\right\},
\end{aligned}
$$

and we have

$$
\begin{gathered}
|a(t)| \leqslant t\left(\frac{1}{2} \mathbb{E}\left|G D^{2}\right|+\mathbb{E}\left|G \tilde{D} D^{\prime}\right|+\mathbb{E}\left|S D^{\prime}\right|\right)=r_{3}|t|=: A_{1}|t|, \\
|b(t)| \leqslant t^{2}\left(t^{-2} \sqrt{\operatorname{Var} \mathbb{E}^{W}\left\{G\left(e^{i t D}-1-i t D\right)\right\}}+t^{-1} \sqrt{\operatorname{Var} \mathbb{E}^{W}\left\{G \tilde{D}\left(e^{i t D^{\prime}}-1\right)\right\}}\right. \\
\left.+t^{-1} \sqrt{\operatorname{Var} \mathbb{E}^{W}\left\{S\left(e^{i t D^{\prime}}-1\right)\right\}}\right)=r_{4} t^{2}=: B_{2} t^{2} .
\end{gathered}
$$

The second claim now follows again from Lemma 2.3 after some straightforward simplifications.

\section{PROOF OF THEOREM 1.1}

We first need some technical results. Recall that a collection of random variables $X=$ $\left(X_{1}, \ldots, X_{n}\right)$ is said to be associated, if for any two coordinate-wise non-decreasing functions $f, g: \mathbb{R}^{m} \rightarrow \mathbb{R}$, we have

$$
\mathbb{E} f(X) \mathbb{E} g(X) \leqslant \mathbb{E}\{f(X) g(X)\}
$$

whenever the expectations exist. 
Lemma 3.1 (Esary, Proschan and Walkup (1967)). (1) A collection of independent random variables is associated. (2) Non-decreasing functions of associated random variables are associated.

Let $[n]:=\{1, \ldots, n\}$ denote the set of vertex labels in $G(n, p)$. For $1 \leqslant i<j \leqslant n$, let $I_{i j}$ be the indicator that there is an edge between vertices $i$ and $j$. Let

$$
\mathcal{E}=\left\{e=\left\{e_{1}, e_{2}\right\} \subset[n]:|e|=2\right\}, \quad \mathcal{T}=\left\{v=\left\{v_{1}, v_{2}, v_{3}\right\} \subset[n]:|v|=3\right\} ;
$$

the first set represent the set of pairs of vertices and the second the set of triples of vertices. We will assume throughout that, in the representations $e=\left\{e_{1}, e_{2}\right\}$ and $v=$ $\left\{v_{1}, v_{2}, v_{3}\right\}$, the elements appear in ascending order; we will also use the notations $I_{e}$ and $I_{e_{1} e_{2}}$ interchageably. For each $v \in \mathcal{T}$, let $X_{v}=I_{v_{1} v_{2}} I_{v_{1} v_{3}} I_{v_{2} v_{3}}-p^{3}$ be the centred indicator that there is a triangle between the vertices in $v$. For concrete indices, such as $v=\{1,2,3\}$, we will simply write $X_{123}$ instead of $X_{\{1,2,3\}}$.

As a direct consequence from Lemma 3.1 we have the following.

Corollary 3.2. (1) For any $E \subset \mathcal{E}$, the random variables $\left(X_{v}\right)_{v \in \mathcal{T}}$, given $\left(I_{e}\right)_{e \in E}$, are associated. (2) For any $T_{1}, T_{2} \subset \mathcal{T}$ and any $E \subset \mathcal{E}$ the random variables $\sum_{v \in T_{1}} X_{v}$ and $\sum_{v \in T_{2}} X_{v}$, given $\left(I_{e}\right)_{e \in E}$, are associated.

Lemma 3.3 (Newman (1980, Lemma 3)). If $U$ and $V$ are associated random variables, then, for any complex valued functions $f$ and $g$,

$$
\operatorname{Cov}(f(U), g(V)) \leqslant\left\|f^{\prime}\right\|\left\|g^{\prime}\right\| \operatorname{Cov}(U, V),
$$

where for complex valued $f,\|f\|=\sup _{x \in \mathbb{R}}|f(x)|$.

Lemma 3.4 (Conditional covariance formula). For any complex-valued random variables $U$ and $V$ we have

$$
\operatorname{Cov}(U, V)=\mathbb{E} \operatorname{Cov}^{\mathcal{F}}(U, V)+\operatorname{Cov}\left(\mathbb{E}^{\mathcal{F}} U, \mathbb{E}^{\mathcal{F}} V\right) .
$$

Lemma 3.5. For any complex-valued random variables $U, V, \tilde{V}, U^{\prime}, V^{\prime}$ and $\tilde{V}^{\prime}$, we have

$$
\left|\operatorname{Cov}\left(U V, U^{\prime} V^{\prime}\right)\right| \leqslant\left|\operatorname{Cov}\left(U \tilde{V}, U^{\prime} \tilde{V}^{\prime}\right)\right|+R
$$

where

$$
\begin{aligned}
R= & \mathbb{E}\left|U(V-\tilde{V}) U^{\prime} V^{\prime}\right|+\mathbb{E}|U(V-\tilde{V})| \mathbb{E}\left|U^{\prime} V^{\prime}\right| \\
& +\mathbb{E}\left|U \tilde{V}^{\prime} U^{\prime}\left(V^{\prime}-\tilde{V}^{\prime}\right)\right|+\mathbb{E}|U \tilde{V}| \mathbb{E}\left|U^{\prime}\left(V^{\prime}-\tilde{V}^{\prime}\right)\right|
\end{aligned}
$$

Proof. Using the linearity properties of the covariance function, we have

$$
\operatorname{Cov}\left(U V, U^{\prime} V^{\prime}\right)=\operatorname{Cov}\left(U \tilde{V}, U^{\prime} \tilde{V}^{\prime}\right)+\operatorname{Cov}\left(U(V-\tilde{V}), U^{\prime} V^{\prime}\right)+\operatorname{Cov}\left(U \tilde{V}, U^{\prime}\left(V^{\prime}-\tilde{V}^{\prime}\right)\right),
$$

from which the claim easily follows.

Lemma 3.6. There exist universal constants $c$ and $C$ such that for all $n \geqslant 3$ and for all $0<p<1$,

$$
c s^{2}(n, p) \leqslant \operatorname{Var} T \leqslant C s^{2}(n, p),
$$

where

$$
s^{2}(n, p)= \begin{cases}n^{4}(1-p) & \text { if } 1 / 2<p<1, \\ n^{4} p^{5} & \text { if } n^{-1 / 2}<p \leqslant 1 / 2, \\ n^{3} p^{3} & \text { if } 0<p \leqslant n^{-1 / 2} .\end{cases}
$$


Proof. This follows easily from

$$
\operatorname{Var} T=\left(\begin{array}{l}
n \\
3
\end{array}\right)\left(p^{3}(1-p)^{3}+3(n-3) p^{5}(1-p)\right)=\left(\begin{array}{c}
n \\
3
\end{array}\right) p^{3}(1-p)\left(1+p+p^{2}+3(n-3) p^{2}\right) .
$$

For the following lemmas, we need some notation. Let $v_{1}, \ldots, v_{k} \in \mathcal{T}$; define

$$
M\left(v_{1}, \ldots, v_{k}\right)=\bigcup_{i=1}^{k}\left(\left\{v_{i, 1}, v_{i, 2}\right\} \cup\left\{v_{i, 1}, v_{i, 3}\right\} \cup\left\{v_{i, 2}, v_{i, 3}\right\}\right) \subset \mathcal{E} ;
$$

this represents the set of unique independent edge indicators induced by a collection of vertices. Let

$$
\nu_{v}:=\{u \in \mathcal{T}:|u \cap v| \geqslant 2\}, \quad Y_{v}:=\sum_{u \in \nu_{v}} X_{u} ;
$$

this represents the triangle indicators that share at least one edge with $X_{v}$ and are thus not independent of $X_{v}$. Moreover, for each $w \in \nu_{v}$, let

$$
\nu_{v, w}=\nu_{u} \cup \nu_{u}, \quad Y_{v, w}:=\sum_{u \in \nu_{v}} X_{v, w} ;
$$

this represents the triangle indicators that share at least one edge with either $X_{v}$ or $X_{w}$ (or both), and are thus not independent of $\left(X_{v}, X_{w}\right)$.

Lemma 3.7. Let $k \geqslant 1$, and let $v_{1}, \ldots, v_{k} \in \mathcal{T}$. Then

$$
\mathbb{E}\left|X_{v_{1}} \cdots X_{v_{k}}\right| \leqslant C(k) \min \left\{1-p, p^{m}\right\}
$$

where $m=\left|M\left(v_{1}, \ldots, v_{k}\right)\right|$. In addition, let $w_{1}, \ldots, w_{l} \in \mathcal{T}, l \geqslant 1$. Then

$$
\operatorname{Cov}\left(X_{v_{1}} \cdots X_{v_{k}}, X_{w_{1}} \cdots X_{w_{l}}\right) \leqslant C(k, l) \min \left\{1-p, p^{m}\right\}
$$

where $m=\left|M\left(v_{1}, \ldots, v_{k}, w_{1} \ldots, w_{l}\right)\right|$.

Proof. Since all $X_{v_{i}}$ are bounded by one, we have, on the one hand,

$$
\mathbb{E}\left|X_{v_{1}} \cdots X_{v_{k}}\right| \leqslant \mathbb{E}\left|X_{v_{1}}\right|=2(1-p) p^{3}\left(1+p+p^{2}\right) \leqslant 6(1-p) .
$$

On the other hand,

$$
\mathbb{E}\left|X_{v_{1}} \cdots X_{v_{k}}\right| \leqslant \sum_{A \subset[k]} \mathbb{E}\left\{\prod_{i \in A} I_{v_{i, 1} v_{i, 2}} I_{v_{i, 1} v_{i, 3}} I_{v_{i, 2} v_{i, 3}}\right\} \prod_{i \in A^{c}} p^{3} \leqslant 2^{k} p^{m} .
$$

The second part of the lemma is a straightforward consequence of the first part.

Lemma 3.8. Let $v, v^{\prime} \in \mathcal{T}$, and let $w \in \nu_{v}$ and $w^{\prime} \in \nu_{v^{\prime}}$. Let $f$ and $g$ be differentiable, complex-valued functions with $f(0)=g(0)=0$. Then

$$
\begin{aligned}
\operatorname{Cov}\left(X_{v} X_{w} f\left(Y_{v}\right), X_{v^{\prime}} X_{w^{\prime}} g\left(Y_{v^{\prime}}\right)\right) & \vee \operatorname{Cov}\left(X_{v} X_{w} f\left(Y_{v, w}\right), X_{v^{\prime}} X_{w^{\prime}} g\left(Y_{v^{\prime}, w^{\prime}}\right)\right) \\
& \leqslant C\left\|f^{\prime}\right\|\left\|g^{\prime}\right\| \min \left\{n^{2}(1-p), p^{m}+n p^{m+2}+n^{2} p^{m+4}\right\},
\end{aligned}
$$

where $m=\left|M\left(v, w, v^{\prime}, w^{\prime}\right)\right|$. 
Proof. We only prove the bound for $\operatorname{Cov}\left(X_{v} X_{w} f\left(Y_{v, w}\right), X_{v^{\prime}} X_{w^{\prime}} g\left(Y_{v^{\prime}, w^{\prime}}\right)\right)$, as the proof for $\operatorname{Cov}\left(X_{v} X_{w} f\left(Y_{v}\right), X_{v^{\prime}} X_{w^{\prime}} g\left(Y_{v^{\prime}}\right)\right)$ is essentially the same. Since $|f(x)| \leqslant\left\|f^{\prime}\right\||x|$ and $|g(x)| \leqslant$ $\| g^{\prime}|||x|$,

$$
\begin{aligned}
& \operatorname{Cov}\left(X_{v} X_{w} f\left(Y_{v, w}\right), X_{v^{\prime}} X_{w^{\prime}} g\left(Y_{v^{\prime}, w^{\prime}}\right)\right) \\
& \quad \leqslant\left\|f^{\prime}\right\|\left\|g^{\prime}\right\|\left(\mathbb{E}\left|X_{v} X_{w} Y_{v, w} X_{v^{\prime}} X_{w^{\prime}} Y_{v^{\prime}, w^{\prime}}\right|+\mathbb{E}\left|X_{v} X_{w} Y_{v, w}\right| \mathbb{E}\left|X_{v^{\prime}} X_{w^{\prime}} Y_{v^{\prime}, w^{\prime}}\right|\right) .
\end{aligned}
$$

We only show how to bound the first expectation above, since the second expression, the product of expectations, can be bounded analogously. From Lemma 3.7, the bound $C n^{2}(1-$ $p)$ is straightforward since both $Y_{v, w}$ and $Y_{v^{\prime}, w^{\prime}}$ contain order $n$ summands. Now, let $u \in$ $\nu_{v, w}$ and $u^{\prime} \in \nu_{v^{\prime}, w^{\prime}}$; note that $\left|\left(u \cup u^{\prime}\right) \backslash\left(v \cup w \cup v^{\prime} \cup w^{\prime}\right)\right| \leqslant 2$, since $u$ is sharing at least two indices with $v$ or $w$, and $u^{\prime}$ is sharing at least two vertices with $v^{\prime}$ or $w^{\prime}$. We can thus distinguish three cases.

1. " $\left(u \cup u^{\prime}\right) \backslash\left(v \cup w \cup v^{\prime} \cup w^{\prime}\right) \mid=0$ ". This can happen at most $2\left(\begin{array}{l}8 \\ 3\end{array}\right)$ times. In this case, $M\left(v, w, v^{\prime}, w^{\prime}, u, u^{\prime}\right) \geqslant M\left(v, w, v^{\prime}, w^{\prime}\right)=m$, and so, by Lemma 3.7 .

$$
\mathbb{E}\left|X_{v} X_{w} X_{u} X_{v^{\prime}} X_{w^{\prime}} Y_{u^{\prime}}\right| \leqslant C p^{m} .
$$

2. " $\left(u \cup u^{\prime}\right) \backslash\left(v \cup w \cup v^{\prime} \cup w^{\prime}\right) \mid=1$ "; this can happen at most order $n$ times. In this case, $M\left(v, w, v^{\prime}, w^{\prime}, u, u^{\prime}\right) \geqslant M\left(v, w, v^{\prime}, w^{\prime}\right)+2$, and so, by Lemma 3.7,

$$
\mathbb{E}\left|X_{v} X_{w} X_{u} X_{v^{\prime}} X_{w^{\prime}} Y_{u^{\prime}}\right| \leqslant C p^{m+2} .
$$

3. " $\left|\left(u \cup u^{\prime}\right) \backslash\left(v \cup w \cup v^{\prime} \cup w^{\prime}\right)\right|=2$ "; this can happen at most order $n^{2}$ times. In this case, $M\left(v, w, v^{\prime}, w^{\prime}, u, u^{\prime}\right) \geqslant M\left(v, w, v^{\prime}, w^{\prime}\right)+4$, and so, by Lemma 3.7,

$$
\mathbb{E}\left|X_{v} X_{w} X_{u} X_{v^{\prime}} X_{w^{\prime}} Y_{u^{\prime}}\right| \leqslant C p^{m+4} .
$$

Putting the estimates together yields the claim.

Lemma 3.9. Let $v, v^{\prime} \in \mathcal{T}$, such that $\left|v \cap v^{\prime}\right|=1$, and let $w \in \nu_{v}$ and $w^{\prime} \in \nu_{v^{\prime}}$ be such that $\left.\mid\left(w \cap w^{\prime}\right) \backslash\left(v \cap v^{\prime}\right)\right) \mid=0$. Let $f$ and $g$ be differentiable, complex-valued functions with $f(0)=g(0)=0$. Then

$$
\begin{aligned}
\operatorname{Cov}\left(X_{v} X_{w} f\left(Y_{v}\right), X_{v^{\prime}} X_{w^{\prime}} g\left(Y_{v^{\prime}}\right)\right) \vee \operatorname{Cov}\left(X_{v} X_{w} f\left(Y_{v, w}\right), X_{v^{\prime}} X_{w^{\prime}} g\left(Y_{v^{\prime}, w^{\prime}}\right)\right) \\
\leqslant C\left\|f^{\prime}\right\|\left\|g^{\prime}\right\| \min \left\{n(1-p), p^{m+1}+n p^{m+3}\right\},
\end{aligned}
$$

where $m=\left|M\left(v, w, v^{\prime}, w^{\prime}\right)\right|$.

Proof. As in the proof of Lemma 3.8, we only consider $\operatorname{Cov}\left(X_{v} X_{w} f\left(Y_{v, w}\right), X_{v^{\prime}} X_{w^{\prime}} g\left(Y_{v^{\prime}, w^{\prime}}\right)\right)$. Throughout the proof, we suppress the dependence on $v, w, v^{\prime}$ and $w^{\prime}$ in many places, since they are fixed. Define the sets

$$
\begin{aligned}
\eta & =\left\{u \in \nu_{v, w} \backslash\{v, w\}:\left|u \cap\left(v^{\prime} \cup w^{\prime}\right)\right|=1\right\}, \\
\eta^{\prime} & =\left\{u \in \nu_{v^{\prime}, w^{\prime}} \backslash\left\{v^{\prime}, w^{\prime}\right\}:|u \cap(v \cup w)|=1\right\}
\end{aligned}
$$

(note that, under the conditions imposed on $v, w, v^{\prime}$ and $w^{\prime}$, we cannot have $\left|u \cap\left(v^{\prime} \cup w^{\prime}\right)\right|>$ 1). The set $\eta$ represents the set of indices of those triangle indicators in $\nu_{v, w}$ which are not equal to $v$ and $w$ and which have one vertex in the set $v^{\prime} \cup w^{\prime}$, and likewise, the set $\eta^{\prime}$ represents the set of indices of those triangle indicators in $\nu_{v^{\prime}, w^{\prime}}$ which are not equal 
to $v^{\prime}$ and $w^{\prime}$ and which have one vertex in the set $v \cup w$. It is important to note that for each $u \in \eta \cup \eta^{\prime}$, we have

$$
\left|M\left(v, w, v^{\prime}, w^{\prime}, u\right)\right| \geqslant\left|M\left(v, w, v^{\prime}, w^{\prime}\right)\right|+1=m+1 .
$$

Now, let

$$
Z_{v, w}=\sum_{u \in \eta} X_{u}, \quad Z_{v^{\prime}, w^{\prime}}=\sum_{u \in \eta^{\prime}} X_{u}
$$

and let

$$
\tilde{Y}_{v, w}=Y_{v}-Z_{v}, \quad \tilde{Y}_{v^{\prime}, w^{\prime}}=Y_{v^{\prime}}-Z_{v^{\prime}} .
$$

The sum $\tilde{Y}_{v, w}$ consists of those centred triangle indicators which are $(i)$ equal to $X_{v}$ or $X_{w}$, or (ii) composed entirely of vertices from $v \cup w$, or (iii) share one edge with $X_{v}$ or $X_{w}$, but whose third vertex is not in $v \cup w \cup v^{\prime} \cup w^{\prime}$ (and the analogous statement holds for $\tilde{Y}_{v^{\prime}, w^{\prime}}$ with cases $\left(i^{\prime}\right),\left(i i^{\prime}\right)$ and $\left.\left(i i i^{\prime}\right)\right)$. Note that if $X_{u}$ is a summand in $\tilde{Y}_{v, w}$ from (iii) above, and if $X_{u^{\prime}}$ is a summand in $\tilde{Y}_{v^{\prime}, w^{\prime}}$ from $\left(i i i^{\prime}\right)$, and if $u$ and $u^{\prime}$ are such that the respective third vertex is equal, then

$$
\left|M\left(v, w, v^{\prime}, w^{\prime}, u, u^{\prime}\right)\right| \geqslant\left|M\left(v, w, v^{\prime}, w^{\prime}\right)\right|+3=m+3 .
$$

We now apply Lemma 3.5 with

$$
\begin{aligned}
& U=X_{v} X_{w}, \\
& V=f\left(Y_{v, w}\right), \\
& \tilde{V}=f\left(\tilde{Y}_{v, w}\right), \\
& U^{\prime}=X_{v^{\prime}} X_{w^{\prime}}, \\
& V^{\prime}=f\left(Y_{v^{\prime}, w^{\prime}}\right), \\
& \tilde{V}^{\prime}=f\left(\tilde{Y}_{v^{\prime}, w^{\prime}}\right) \text {. }
\end{aligned}
$$

Note that, since $|f(x)| \leqslant\left\|f^{\prime}\right\||x|$ and $|g(x)| \leqslant\left\|g^{\prime}\right\||x|$, we have

$$
|V-\tilde{V}| \leqslant\left\|f^{\prime}||\left|Z_{v, w}\right|, \quad\left|V^{\prime}-\tilde{V}^{\prime}\right| \leqslant\right\| g^{\prime} \|\left|Z_{v^{\prime}, w^{\prime}}\right| .
$$

We obtain

$$
\left|\operatorname{Cov}\left(X_{v} X_{w} f\left(Y_{v, w}\right), X_{v^{\prime}} X_{w^{\prime}} g\left(Y_{v^{\prime}, w^{\prime}}\right)\right)\right| \leqslant\left|\operatorname{Cov}\left(X_{v} X_{w} f\left(\tilde{Y}_{v, w}\right), X_{v^{\prime}} X_{w^{\prime}} g\left(\tilde{Y}_{v^{\prime}, w^{\prime}}\right)\right)\right|+R,
$$

where

$$
\begin{aligned}
R \leqslant & \mathbb{E}\left|X_{v} X_{w} Z_{v, w} X_{v^{\prime}} X_{w^{\prime}} Y_{v^{\prime}, w^{\prime}}\right|+\mathbb{E}\left|X_{v} X_{w} Z_{v, w}\right| \mathbb{E}\left|X_{v^{\prime}} X_{w^{\prime}} Y_{v^{\prime}, w^{\prime}}\right| \\
& +\mathbb{E}\left|X_{v} X_{w} \tilde{Y}_{v, w} X_{v^{\prime}} X_{w^{\prime}} Z_{v^{\prime}, w^{\prime}}\right|+\mathbb{E}\left|X_{v} X_{w} \tilde{Y}_{v, w}\right| \mathbb{E}\left|X_{v^{\prime}} X_{w^{\prime}} Z_{v^{\prime}, w^{\prime}}\right|
\end{aligned}
$$

From this, (3.4), (3.5) and Lemma 3.7, it is not difficult to see that

$$
R \leqslant C \min \left\{n(1-p), p^{m+1}+n p^{m+3}\right\} .
$$

Now, let

$$
M^{*}(v, w)=\{u \in \mathcal{T}: u \subset(v \cup w)\}
$$

and define the $\sigma$-algebras

$$
\mathcal{F}_{1}=\sigma\left(I_{e} ; e \in M^{*}(v, w)\right), \quad \mathcal{F}_{2}=\sigma\left(I_{e} ; e \in M^{*}\left(v^{\prime}, w^{\prime}\right)\right) ;
$$

note that $X_{v} X_{w}$ is $\mathcal{F}_{1}$-measurable and $X_{v^{\prime}} X_{w^{\prime}}$ is $\mathcal{F}_{2}$-measurable, and that $\mathcal{F}_{1}$ and $\mathcal{F}_{2}$ are independent of each other. With $\mathcal{F}=\sigma\left(\mathcal{F}_{1}, \mathcal{F}_{2}\right)$, we apply Lemma 3.4 and obtain

$$
\begin{aligned}
\operatorname{Cov}\left(X_{v} X_{w} f\left(\tilde{Y}_{v, w}\right), X_{v^{\prime}} X_{w^{\prime}} g\left(\tilde{Y}_{v^{\prime}, w^{\prime}}\right)\right)= & \operatorname{Cov}\left(X_{v} X_{w} \mathbb{E}^{\mathcal{F}} f\left(\tilde{Y}_{v, w}\right), X_{v^{\prime}} X_{w^{\prime}} \mathbb{E}^{\mathcal{F}} g\left(\tilde{Y}_{v^{\prime}, w^{\prime}}\right)\right) \\
& +\mathbb{E} \operatorname{Cov}^{\mathcal{F}}\left(X_{v} X_{w} f\left(\tilde{Y}_{v, w}\right), X_{v^{\prime}} X_{w^{\prime}} g\left(\tilde{Y}_{v^{\prime}, w^{\prime}}\right)\right) \\
= & : a_{1}+a_{2} .
\end{aligned}
$$


Now, by construction of $\tilde{Y}_{v, w}$ and $\tilde{Y}_{v^{\prime}, w^{\prime}}$, we have $\mathbb{E}^{\mathcal{F}} f\left(\tilde{Y}_{v, w}\right)=\mathbb{E}^{\mathcal{F}_{1}} f\left(\tilde{Y}_{v, w}\right)$ and $\mathbb{E}^{\mathcal{F}} g\left(\tilde{Y}_{v^{\prime}, w^{\prime}}\right)=$ $\mathbb{E}^{\mathcal{F}_{2}} g\left(\tilde{Y}_{v^{\prime}, w^{\prime}}\right)$, from which $a_{1}=0$ is immediate. In order to bound $a_{2}$, we write

$$
\operatorname{Cov}^{\mathcal{F}}\left(X_{v} X_{w} f\left(\tilde{Y}_{v, w}\right), X_{v^{\prime}} X_{w^{\prime}} g\left(\tilde{Y}_{v^{\prime}, w^{\prime}}\right)\right)=X_{v} X_{w} X_{v^{\prime}} X_{w^{\prime}} \operatorname{Cov}^{\mathcal{F}}\left(f\left(\tilde{Y}_{v, w}\right), g\left(\tilde{Y}_{v^{\prime}, w^{\prime}}\right)\right) .
$$

Using Corollary 3.2 and Lemma 3.3, we have

$$
\left|\operatorname{Cov}^{\mathcal{F}}\left(f\left(\tilde{Y}_{v, w}\right), g\left(\tilde{Y}_{v^{\prime}, w^{\prime}}\right)\right)\right| \leqslant\left\|f^{\prime}\right\|\left\|g^{\prime}\right\| \operatorname{Cov}^{\mathcal{F}}\left(\tilde{Y}_{v, w}, \tilde{Y}_{v^{\prime}, w^{\prime}}\right) .
$$

We now can write

$$
\begin{aligned}
\operatorname{Cov}^{\mathcal{F}}\left(\tilde{Y}_{v, w}, \tilde{Y}_{v^{\prime}, w^{\prime}}\right)= & \sum_{\substack{e \in M(v, w), e^{\prime} \in M\left(v^{\prime}, w^{\prime}\right)}} \sum_{i \in[n] \backslash\left(v \cup w \cup v^{\prime} \cup w^{\prime}\right)} \operatorname{Cov}^{\mathcal{F}}\left(X_{e_{1} e_{2} i}, X_{e_{1}^{\prime} e_{2}^{\prime} i}\right) \\
& +\sum_{\substack{e \in M(v, w), e^{\prime} \in M\left(v^{\prime}, w^{\prime}\right)}} \sum_{\substack{i, j \in[n] \backslash\left(v \cup w \cup v^{\prime} \cup w^{\prime}\right), i \neq j}} \operatorname{Cov}^{\mathcal{F}}\left(X_{e_{1} e_{2} i}, X_{e_{1}^{\prime} e_{2}^{\prime} j}\right) .
\end{aligned}
$$

From the assertions we conclude that $e \in M(v, w)$ and $e^{\prime} \in M\left(v^{\prime}, w^{\prime}\right)$ implies $e \neq e^{\prime}$. Hence, we can use independence to find that the covariances in the second sum all vanish, and moreover, that $M\left(v, w, v^{\prime}, w^{\prime},\left\{e_{1}, e_{2}, i\right\},\left\{e_{1}^{\prime}, e_{2}^{\prime}, i\right\}\right) \geqslant M\left(v, w, v^{\prime}, w^{\prime}\right)+3$. Thus, Lemma 3.7 yields

$$
\begin{array}{r}
a_{2} \leqslant \mathbb{E}\left|X_{v} X_{w} X_{v^{\prime}} X_{w^{\prime}} \sum_{\substack{e \in M(v, w), e^{\prime} \in M\left(v^{\prime}, w^{\prime}\right)}} \sum_{i \in[n] \backslash\left(v \cup w \cup v^{\prime} \cup w^{\prime}\right)} \operatorname{Cov}^{\mathcal{F}}\left(X_{e_{1} e_{2}}, X_{e_{1}^{\prime} e_{2}^{\prime} i}\right)\right| \\
\leqslant C \min \left\{n(1-p), n p^{m+3}\right\} .
\end{array}
$$

Collecting all the estimates gives the final bound.

Lemma 3.10. Let $v, v^{\prime} \in \mathcal{T}$ be such that $\left|v \cap v^{\prime}\right|=0$, and let $w \in \nu_{v}$ and $w^{\prime} \in \nu_{v^{\prime}}$ be such that $\left|(v \cup w) \cap\left(v^{\prime} \cup w^{\prime}\right)\right|=0$. Let $f$ and $g$ be differentiable, complex-valued functions with $f(0)=g(0)=0$. Then

$$
\begin{aligned}
\operatorname{Cov}\left(X_{v} X_{w} f\left(Y_{v}\right), X_{v^{\prime}} X_{w^{\prime}} g\left(Y_{v^{\prime}}\right)\right) \vee \operatorname{Cov}\left(X_{v} X_{w} f\left(Y_{v, w}\right), X_{v^{\prime}} X_{w^{\prime}} g\left(Y_{v^{\prime}, w^{\prime}}\right)\right) \\
\leqslant C\left\|f^{\prime}\right\|\left\|g^{\prime}\right\| \min \left\{1-p, p^{m+3}\right\},
\end{aligned}
$$

where $m=\left|M\left(v, w, v^{\prime}, w^{\prime}\right)\right|$.

Proof. As in Lemma 3.8, we only consider $\operatorname{Cov}\left(X_{v} X_{w} f\left(Y_{v, w}\right), X_{v^{\prime}} X_{w^{\prime}} g\left(Y_{v^{\prime}, w^{\prime}}\right)\right)$. Let $\mathcal{F}_{1}$, $\mathcal{F}_{2}$ and $\mathcal{F}$ be as in (3.7). Applying Lemma 3.4,

$$
\begin{aligned}
\operatorname{Cov}\left(X_{v} X_{w} f\left(Y_{v, w}\right), X_{v^{\prime}} X_{w^{\prime}} g\left(Y_{v^{\prime}, w^{\prime}}\right)\right)= & \operatorname{Cov}\left(X_{v} X_{w} \mathbb{E}^{\mathcal{F}} f\left(Y_{v, w}\right), X_{v^{\prime}} X_{w^{\prime}} \mathbb{E}^{\mathcal{F}} g\left(Y_{w, w^{\prime}}\right)\right) \\
& +\mathbb{E}\left\{X_{v} X_{w} X_{v^{\prime}} X_{w^{\prime}} \operatorname{Cov}^{\mathcal{F}}\left(f\left(Y_{v, w}\right), g\left(Y_{v^{\prime}, w^{\prime}}\right)\right)\right\} \\
= & : a_{1}+a_{2} .
\end{aligned}
$$

Now, since $\mathbb{E}^{\mathcal{F}} f\left(Y_{v, w}\right)=\mathbb{E}^{\mathcal{F}_{1}} f\left(Y_{v, w}\right)$ and $\mathbb{E}^{\mathcal{F}} f\left(Y_{v^{\prime}, w^{\prime}}\right)=\mathbb{E}^{\mathcal{F}_{2}} f\left(Y_{v^{\prime}, w^{\prime}}\right)$, we have $a_{1}=0$. By Corollary 3.2 and Lemma 3.3 .

$$
\left|\operatorname{Cov}^{\mathcal{F}}\left(f\left(Y_{v, w}\right), g\left(Y_{v^{\prime}, w^{\prime}}\right)\right)\right| \leqslant C\left\|f^{\prime}\right\|\left\|g^{\prime}\right\| \operatorname{Cov}^{\mathcal{F}}\left(Y_{v, w}, Y_{v^{\prime}, w^{\prime}}\right) .
$$


We can write

$$
\begin{aligned}
\operatorname{Cov}^{\mathcal{F}}\left(Y_{v, w}, Y_{v^{\prime}, w^{\prime}}\right)= & \sum_{\substack{e \in M(v, w), e^{\prime} \in M\left(v^{\prime}, w^{\prime}\right)}} \sum_{\substack{i \in\left(v^{\prime} \cup w^{\prime}\right), j \in(v \cup w)}} \operatorname{Cov}^{\mathcal{F}}\left(X_{e_{1} e_{2}}, X_{e_{1}^{\prime} e_{2}^{\prime} j}\right) \\
& +\sum_{\substack{e \in M(v, w), e^{\prime} \in M\left(v^{\prime}, w^{\prime}\right)}} \sum_{\substack{i, j \in\left[\left(v^{\prime} \cup w^{\prime}\right) \text { or } \\
j \notin(v \cup w)\right.}} \operatorname{Cov}^{\mathcal{F}}\left(X_{e_{1} e_{2} i}, X_{e_{1}^{\prime} e_{2}^{\prime} j}\right) .
\end{aligned}
$$

From the assertions we conclude that $e \in M(v, w)$ and $e^{\prime} \in M\left(v^{\prime}, w^{\prime}\right)$ implies $\left|e \cap e^{\prime}\right|=0$. Hence, we can use independence to find that the covariances in the second sum all vanish. For the first sum, note that $M\left(v, w, v^{\prime}, w^{\prime},\left\{e_{1}, e_{2}, i\right\},\left\{e_{1}^{\prime}, e_{2}^{\prime}, j\right\}\right) \geqslant M\left(v, w, v^{\prime}, w^{\prime}\right)+3$. Thus, Lemma 3.7 yields

$$
\begin{aligned}
a_{2} \leqslant \mathbb{E}\left|X_{v} X_{w} X_{v^{\prime}} X_{w^{\prime}} \sum_{\substack{e \in M(v, w), e^{\prime} \in M\left(v^{\prime}, w^{\prime}\right)}} \sum_{\substack{i \in\left(v^{\prime} \cup w^{\prime}\right), j \in(v \cup w)}} \operatorname{Cov}^{\mathcal{F}}\left(X_{e_{1} e_{2} i}, X_{e_{1}^{\prime} e_{2}^{\prime} j}\right)\right| & \\
& \leqslant C \min \left\{1-p, p^{m+3}\right\} .
\end{aligned}
$$

Collecting all the estimates gives the final bound.

Lemma 3.11. Let $v, v^{\prime} \in \mathcal{T}$ be such that $\left|v \cap v^{\prime}\right|=0$, and let $w \in \nu_{v}$ and $w^{\prime} \in \nu_{v^{\prime}}$ be such that $\left|(v \cup w) \cap\left(v^{\prime} \cup w^{\prime}\right)\right|=1$. Let $f$ and $g$ be differentiable, complex-valued functions with $f(0)=g(0)=0$. Then

$$
\begin{aligned}
\operatorname{Cov}\left(X_{v} X_{w} f\left(Y_{v}\right), X_{v^{\prime}} X_{w^{\prime}} g\left(Y_{v^{\prime}}\right)\right) \vee \operatorname{Cov}\left(X_{v} X_{w} f\left(Y_{v, w}\right), X_{v^{\prime}} X_{w^{\prime}} g\left(Y_{v^{\prime}, w^{\prime}}\right)\right) \\
\leqslant C\left\|f^{\prime}\right\|\left\|g^{\prime}\right\| \min \left\{n(1-p), p^{m+1}+n p^{m+3}\right\},
\end{aligned}
$$

where $m=\left|M\left(v, w, v^{\prime}, w^{\prime}\right)\right|$.

Proof. The proof is similar to that of Lemma 3.9 and therefore ommited.

Proof of Theorem 1.1. Construction of Stein coupling. Our proof is based on a Stein coupling that is equivalent to what was implicitly used by Barbour, Karoński and Ruciński (1989). Let

$$
W=\frac{1}{\sigma} \sum_{v \in \mathcal{T}} X_{v}
$$

be the scaled sum of centred triangle indicator random variables. Let $V$ be an independent random variable, uniformly distributed on $\mathcal{T}$; set

$$
G=-\left(\begin{array}{l}
n \\
3
\end{array}\right) \frac{X_{V}}{\sigma}, \quad W^{\prime}=W-\frac{Y_{V}}{\sigma} .
$$

Using the fact that $W-Y_{v} / \sigma$ and $X_{v}$ are independent, it is straightforward to verify that $\left(W, W^{\prime}, G\right)$ is a Stein coupling, and we have $D=-Y_{V} / \sigma$.

Construction of extended Stein coupling. In order to construct $\tilde{D}, S$ and $W^{\prime \prime}$, recall the definition of $\nu_{v}$ from (3.2); note that $\left|\nu_{v}\right|=3(n-3)+1$ and $Y_{v}=\sum_{u \in \nu_{v}} X_{u}$. Given $V$, let $V^{\prime}$ be a random variable, independent of all else and distributed uniformly on $\nu_{v}$. Set

$$
\tilde{D}:=-\frac{3(n-3)+1}{\sigma} X_{V^{\prime}}
$$


It is straightforward to check that

$$
\mathbb{E}\left\{\tilde{D} \mid\left(I_{e}\right)_{e \in \mathcal{E}}, V\right\}=-\frac{Y_{V}}{\sigma}=D,
$$

which implies that $\mathbb{E}^{W}(G \tilde{D})=\mathbb{E}^{W}(G D)$ since $W$ is measurable with respect to $\left(I_{e}\right)_{e \in \mathcal{E}}$. Let

$$
S:=\left(\begin{array}{l}
n \\
3
\end{array}\right) \frac{3(n-3)+1}{\sigma^{2}} \times \begin{cases}\operatorname{Var} X_{123} & \text { if } V^{\prime}=V, \\
\operatorname{Cov}\left(X_{123}, X_{124}\right) & \text { if } V^{\prime} \neq V,\end{cases}
$$

and it is easy to verify that $\mathbb{E}^{W} S=1$. For $v \in \mathcal{T}$ and $w \in \nu_{v}$, recall the definition of $\nu_{v, w}$ in (3.3); let

$$
Y_{v, w}=\sum_{u \in \nu_{v}, w} X_{u}
$$

and moreover, let

$$
W^{\prime \prime}=W-\frac{Y_{V, V^{\prime}}}{\sigma} .
$$

Using independence between $W-Y_{v, w} / \sigma$ and $X_{v} X_{w}$ it follows that

$$
\begin{aligned}
& \mathbb{E}\left\{G \tilde{D} \mid V, W^{\prime \prime}\right\} \\
& =\left(\begin{array}{c}
n \\
3
\end{array}\right) \frac{1+3(n-3)}{\sigma^{2}} \begin{cases}\mathbb{E}\left\{X_{V}^{2}\right\} & \text { if } V=V^{\prime}, \\
\mathbb{E}\left\{X_{V} X_{V^{\prime}}\right\} & \text { if } V \neq V^{\prime}\end{cases} \\
& =S,
\end{aligned}
$$

which implies that $\mathbb{E}^{W^{\prime \prime}}(G \tilde{D})=\mathbb{E}^{W^{\prime \prime}} S$, and we have

$$
D^{\prime}=-\frac{1}{\sigma} Y_{V, V^{\prime}}
$$

Thus, all the conditions in Theorem 2.1 are satisfies, and it remains to bound $r_{3}$ and $r_{4}$.

Bounding $\boldsymbol{r}_{\mathbf{3}}$. In what follows, $C$ denotes a constant that does not depend on $n$ and $p$, and which can change from line to line. First, write

$$
r_{3}=\frac{1}{2} \mathbb{E}\left|G D^{2}\right|+\mathbb{E}\left|G \tilde{D} D^{\prime}\right|+\mathbb{E}\left|S D^{\prime}\right|=: \frac{1}{2} r_{3,1}+r_{3,2}+r_{3,3} .
$$

Using some obvious symmetries, and bounding terms like $n-1, n-2$ and so forth by $n$, we have

$$
r_{3,1} \leqslant \frac{C n^{3}}{\sigma^{3}} \mathbb{E}\left|X_{V} Y_{V}^{2}\right|=\frac{C n^{3}}{\sigma^{3}} \mathbb{E}\left|X_{123} Y_{123}^{2}\right| .
$$

On the one hand, we have the simple bound $\mathbb{E}\left|X_{123} Y_{123}^{2}\right| \leqslant n^{2} \mathbb{E}\left|X_{123}\right| \leqslant C n^{2}(1-p)$, and on the other hand, by means of Lemma 3.7, it is straightforward to see that

$$
\mathbb{E}\left|X_{123} Y_{123}^{2}\right| \leqslant C\left(p^{3}+n p^{5}+n^{2} p^{7}\right) ;
$$

hence,

$$
r_{3,1} \leqslant \frac{C}{\sigma^{3}} \min \left\{n^{5}(1-p), n^{3} p^{3}+n^{4} p^{5}+n^{5} p^{7}\right\}
$$


Moreover,

$$
\begin{aligned}
r_{3,2} & =\mathbb{E}\left|G \tilde{D} D^{\prime}\right| \\
& \leqslant \frac{C n^{4}}{\sigma^{3}} \mathbb{E}\left|X_{V} X_{V^{\prime}} Y_{V^{\prime} V^{\prime}}\right| \\
& \leqslant \frac{C n^{4}}{\sigma^{3}} \mathbb{E}\left|X_{V}^{2} Y_{V} \mathrm{I}\left[V=V^{\prime}\right]+X_{V} X_{V^{\prime}} Y_{V, V^{\prime}} \mathrm{I}\left[V \neq V^{\prime}\right]\right| \\
& \leqslant \frac{C n^{3}}{\sigma^{3}} \mathbb{E}\left|X_{123}^{2} Y_{123}\right|+\frac{C n^{4}}{\sigma^{3}} \mathbb{E}\left|X_{123} X_{124} Y_{123,124}\right| .
\end{aligned}
$$

On the hand hand, we can bound both $\mathbb{E}\left|X_{123}^{2} Y_{123}\right|$ and $\mathbb{E}\left|X_{123} X_{124} Y_{123,124}\right|$ by $C n(1-p)$. On the other hand, we have $\mathbb{E}\left|X_{123}^{2} Y_{123}\right| \leqslant C\left(p^{3}+n p^{5}\right)$ and $\mathbb{E}\left|X_{123} X_{124} Y_{123,124}\right| \leqslant C\left(p^{5}+\right.$ $n p^{7}$ ) from Lemma 3.7, so that

$$
r_{3,2} \leqslant \frac{C}{\sigma^{3}} \min \left\{n^{5}(1-p), n^{3} p^{3}+n^{4} p^{5}+n^{5} p^{7}\right\} .
$$

Finally,

$$
\begin{aligned}
r_{3,3} & =\mathbb{E}\left|S D^{\prime}\right| \\
& \leqslant \frac{C n^{4}}{\sigma^{3}} \mathbb{E}\left|\mathrm{I}\left[V=V^{\prime}\right] Y_{V} \operatorname{Var}\left(X_{123}\right)+\mathrm{I}\left[V \neq V^{\prime}\right] Y_{V, V^{\prime}} \operatorname{Cov}\left(X_{123}, X_{124}\right)\right| \\
& \leqslant \frac{C n^{3}}{\sigma^{3}} \operatorname{Var}\left(X_{123}\right) \mathbb{E}\left|Y_{123}\right|+\frac{C n^{4}}{\sigma^{3}} \mathbb{E}\left|Y_{123,124}\right| \operatorname{Cov}\left(X_{123}, X_{124}\right) .
\end{aligned}
$$

On the one hand, we can bound both $\operatorname{Var} X_{123}$ and $\operatorname{Cov}\left(X_{123}, X_{124}\right)$ by $C(1-p)$, and both $\mathbb{E}\left|Y_{123}\right|$ and $\mathbb{E}\left|Y_{1234}\right|$ by $n$. On the other hand, we have the bounds $\operatorname{Var} X_{123} \leqslant C p^{3}$, $\operatorname{Cov}\left(X_{123}, X_{124}\right) \leqslant C p^{5}, \mathbb{E}\left|Y_{123}\right| \leqslant C n p^{3}$ and $\mathbb{E}\left|Y_{123,124}\right| \leqslant C n p^{3}$. This yields

$$
r_{3,3} \leqslant \frac{C}{\sigma^{3}} \min \left\{n^{5}(1-p), n^{4} p^{6}+n^{5} p^{8}\right\} .
$$

Combining the bounds on $r_{3,1}, r_{3,2}$ and $r_{3,3}$, we obtain

$$
r_{3} \leqslant \begin{cases}\frac{C n^{5}(1-p)}{\sigma^{3}} & \text { if } 1 / 2<p<1, \\ \frac{C n^{5} p^{7}}{\sigma^{3}} & \text { if } n^{-1 / 2}<p \leqslant 1 / 2, \\ \frac{C n^{3} p^{3}}{\sigma^{3}} & \text { if } 0<p \leqslant n^{-1 / 2} .\end{cases}
$$

For later use, we let $\tilde{r}_{3}$ be equal to the right hand side of (3.9).

Bounding $\boldsymbol{r}_{\mathbf{4}}$. First write

$$
r_{4}=: \sup _{t \neq 0} \frac{r_{4,1}(t)^{1 / 2}}{t^{2}}+\sup _{t \neq 0} \frac{r_{4,2}(t)^{1 / 2}}{|t|}+\sup _{t \neq 0} \frac{r_{4,3}(t)^{1 / 2}}{|t|} .
$$

From now on, we consider $t$ as being fixed and, thus, drop it from our notation. Moreover, instead of conditioning $G\left(e^{i t D}-1-i t D\right)$ on $W$, we may condition on $\left(I_{e}\right)_{e \in \mathcal{E}}$, making the corresponding variances in $r_{4}$ only larger. Now, define the function

$$
\varphi(x)= \begin{cases}\frac{e^{i x}-1-i x}{x} & \text { if } x \neq 0, \\ 0 & \text { if } x=0,\end{cases}
$$


and for later use, note that

$$
|\varphi(x)-\varphi(y)| \leqslant \frac{|x-y|}{2} .
$$

Hence,

$$
\begin{aligned}
r_{4,1} & \leqslant \frac{1}{\sigma^{2}} \operatorname{Var} \sum_{v} X_{v}\left(e^{t Y_{v} / \sigma}-1-i t Y_{v} / \sigma\right) \\
& \leqslant \frac{C t^{2} n^{3}}{\sigma^{4}}\left(r_{4,1,1}+n r_{4,1,2}+n^{2} r_{4,1,3}+n^{3} r_{4,1,4}\right),
\end{aligned}
$$

where

$$
\begin{aligned}
& r_{4,1,1}=\operatorname{Cov}\left(X_{123} Y_{123} \varphi\left(t Y_{123} / \sigma\right), X_{123} Y_{123} \varphi\left(t Y_{123} / \sigma\right)\right), \\
& r_{4,1,2}=\left|\operatorname{Cov}\left(X_{123} Y_{123} \varphi\left(t Y_{123} / \sigma\right), X_{124} Y_{124} \varphi\left(t Y_{124} / \sigma\right)\right)\right|, \\
& r_{4,1,3}=\left|\operatorname{Cov}\left(X_{123} Y_{123} \varphi\left(t Y_{123} / \sigma\right), X_{145} Y_{145} \varphi\left(t Y_{145} / \sigma\right)\right)\right|, \\
& r_{4,1,4}=\left|\operatorname{Cov}\left(X_{123} Y_{123} \varphi\left(t Y_{123} / \sigma\right), X_{456} Y_{456} \varphi\left(t Y_{456} / \sigma\right)\right)\right| .
\end{aligned}
$$

Combining all estimates from Tables 1 to 4, we obtain

$$
r_{4,1} \leqslant \begin{cases}\frac{C t^{4} n^{8}(1-p)}{\sigma^{6}} & \text { if } 1 / 2<p<1, \\ \frac{C t^{4} n^{8} p^{13}}{\sigma^{6}} & \text { if } n^{-1 / 2}<p \leqslant 1 / 2, \\ \frac{C t^{4} n^{3} p^{3}}{\sigma^{6}} & \text { if } 0<p \leqslant n^{-1 / 2} .\end{cases}
$$

We continue to bound $r_{4,2}$; to this end, define

$$
\psi(x)=e^{i x}-1,
$$

and, again for later use, note that

$$
|\psi(x)-\psi(y)| \leqslant|x-y| .
$$

Hence,

$$
\begin{aligned}
r_{4,2} & \leqslant \frac{1}{\sigma^{4}} \operatorname{Var} \sum_{v \in \mathcal{T}} X_{v} \sum_{w \in \eta_{v}} X_{w}\left(e^{t Y_{v, w} / \sigma}-1\right) \\
& \leqslant \frac{C n^{3}}{\sigma^{4}}\left(r_{4,2,1}+n r_{4,2,2}+n^{2} r_{4,2,3}+n^{3} r_{4,2,4}\right)
\end{aligned}
$$

where

$$
\begin{aligned}
& r_{4,2,1}=\operatorname{Cov}\left(X_{123} \sum_{w \in \nu_{123}} X_{w} \psi\left(t Y_{123, w} / \sigma\right), X_{123} \sum_{w \in \nu_{123}} X_{w} \psi\left(t Y_{123, w} / \sigma\right)\right), \\
& r_{4,2,2}=\left|\operatorname{Cov}\left(X_{123} \sum_{w \in \nu_{123}} X_{w} \psi\left(t Y_{123, w} / \sigma\right), X_{124} \sum_{w \in \nu_{124}} X_{w} \psi\left(t Y_{124, w} / \sigma\right)\right)\right|, \\
& r_{4,2,3}=\left|\operatorname{Cov}\left(X_{123} \sum_{w \in \nu_{123}} X_{w} \psi\left(t Y_{123, w} / \sigma\right), X_{145} \sum_{w \in \nu_{145}} X_{w} \psi\left(t Y_{145, w} / \sigma\right)\right)\right|, \\
& r_{4,2,4}=\left|\operatorname{Cov}\left(X_{123} \sum_{w \in \nu_{123}} X_{w} \psi\left(t Y_{123, w} / \sigma\right), X_{456} \sum_{w \in \nu_{456}} X_{w} \psi\left(t Y_{456, w} / \sigma\right)\right)\right| .
\end{aligned}
$$

Combining all estimates from Tables 1 to 4, we obtain

$$
r_{4,2} \leqslant \begin{cases}\frac{C t^{2} n^{8}(1-p)}{\sigma^{6}} & \text { if } 1 / 2<p<1, \\ \frac{C t^{2} n^{8} p^{13}}{\sigma^{6}} & \text { if } n^{-1 / 2}<p \leqslant 1 / 2, \\ \frac{C t^{2} n^{3} p^{3}}{\sigma^{6}} & \text { if } 0<p \leqslant n^{-1 / 2} .\end{cases}
$$


We proceed to bound $r_{4,3}$. Let $\sigma_{v, w}=\operatorname{Cov}\left(X_{v}, X_{w}\right)$, we have

$$
\begin{aligned}
r_{4,3} & \leqslant \frac{1}{\sigma^{4}} \operatorname{Var} \sum_{v \in \mathcal{T}} \sum_{w \in \eta_{v}} \sigma_{v, w}\left(e^{t Y_{v, w} / \sigma}-1\right) \\
& \leqslant \frac{C n^{3}}{\sigma^{4}}\left(r_{4,3,1}+n r_{4,3,2}+n^{2} r_{4,3,3}+n^{3} r_{4,3,4}\right)
\end{aligned}
$$

where

$$
\begin{aligned}
& r_{4,3,1}=\operatorname{Cov}\left(\sum_{w \in \nu_{123}} \sigma_{123, w} \psi\left(t Y_{123, w} / \sigma\right), \sum_{w \in \nu_{123}} \sigma_{123, w} \psi\left(t Y_{123, w} / \sigma\right)\right), \\
& r_{4,3,2}=\left|\operatorname{Cov}\left(\sum_{w \in \nu_{123}} \sigma_{123, w} \psi\left(t Y_{123, w} / \sigma\right), \sum_{w \in \nu_{124}} \sigma_{124, w} \psi\left(t Y_{124, w} / \sigma\right)\right)\right|, \\
& r_{4,3,3}=\left|\operatorname{Cov}\left(\sum_{w \in \nu_{123}} \sigma_{123, w} \psi\left(t Y_{123, w} / \sigma\right), \sum_{w \in \nu_{145}} \sigma_{145, w} \psi\left(t Y_{145, w} / \sigma\right)\right)\right|, \\
& r_{4,3,4}=\left|\operatorname{Cov}\left(\sum_{w \in \nu_{123}} \sigma_{123, w} \psi\left(t Y_{123, w} / \sigma\right), \sum_{w \in \nu_{456}} \sigma_{456, w} \psi\left(t Y_{456, w} / \sigma\right)\right)\right| .
\end{aligned}
$$

Bounds can also be obtained from Tables 1 to 4 , since $\sigma_{v, w} \sigma_{v^{\prime}, w^{\prime}} \leqslant C \min \left\{1-p, p^{m}\right\}$ where $m=\left|M\left(v, w, v^{\prime}, w^{\prime}\right)\right|$, so that bounds on $r_{4,2,1}$ to $r_{4,2,4}$ are also bounds on $r_{4,3,1}$ to $r_{4,3,4}$. Thus,

$$
r_{4,3} \leqslant \begin{cases}\frac{C t^{2} n^{8}(1-p)}{\sigma^{6}} & \text { if } 1 / 2<p<1, \\ \frac{C t^{2} n^{8} p^{13}}{\sigma^{6}} & \text { if } n^{-1 / 2}<p \leqslant 1 / 2, \\ \frac{C t^{2} n^{3} p^{3}}{\sigma^{6}} & \text { if } 0<p \leqslant n^{-1 / 2} .\end{cases}
$$

Putting the estimates together, we obtain

$$
r_{4} \leqslant \begin{cases}\frac{C n^{4}(1-p)^{1 / 2}}{\sigma^{3}} & \text { if } 1 / 2<p<1, \\ \frac{C n^{4} p^{13 / 2}}{\sigma^{3}} & \text { if } n^{-1 / 2}<p \leqslant 1 / 2, \\ \frac{C n^{3 / 2} p^{3 / 2}}{\sigma^{3}} & \text { if } 0<p \leqslant n^{-1 / 2} .\end{cases}
$$

From Lemma 3.6,

$$
\sigma^{2} \geqslant \begin{cases}C n^{4}(1-p) & \text { if } 1 / 2<p<1 \\ C n^{4} p^{5} & \text { if } n^{-1 / 2}<p \leqslant 1 / 2 \\ C n^{3} p^{3} & \text { if } 0<p \leqslant n^{-1 / 2}\end{cases}
$$

Applying Theorem 2.1, the final bound follows.

\subsection{Covariance estimates}

In the following tables, we provide bounds necessary for the proof of Theorem 1.1, We illustrate how to read the tables by means of $r_{4,1,3}$ from the proof of Theorem 1.1. We have

$$
r_{4,1,3}=\left|\operatorname{Cov}\left(X_{123} Y_{123} \varphi\left(t Y_{123} / \sigma\right), X_{145} Y_{145} \varphi\left(t Y_{145} / \sigma\right)\right)\right|
$$


expanding the covariance over $Y_{123}$ and $Y_{145}$, we have

$$
r_{4,1,3} \leqslant \sum_{u \in \nu_{123}} \sum_{u^{\prime} \in \nu_{145}}\left|\operatorname{Cov}\left(X_{123} X_{u} \varphi\left(t Y_{123} / \sigma\right), X_{145} X_{u^{\prime}} \varphi\left(t Y_{145} / \sigma\right)\right)\right| .
$$

Table 3 now gives bound on these covariance terms for all possible combinations of $u$ and $u^{\prime}$ (modulo symmetries). The solid thick line represents the variable $X_{123}$, the dashed thick line the variable $X_{u}$, the solid thin line the variable $X_{145}$ and the dashed thin line the variable $X_{u^{\prime}}$. For example, for $u=\{2,3,4\}$ and $u^{\prime}=\{4,5,6\}$ (15th row in Table 3) we find that

$$
\begin{aligned}
\left|\operatorname{Cov}\left(X_{123} X_{234} \varphi\left(t Y_{123} / \sigma\right), X_{145} X_{456} \varphi\left(t Y_{145} / \sigma\right)\right)\right| & \\
& \leqslant \frac{C t^{2}}{\sigma^{2}} \min \left\{n^{2}(1-p), p^{9}+n p^{11}+n^{2} p^{13}\right\} .
\end{aligned}
$$

This covariance term appears order $n$ times in $r_{4,1,3}$, since vertex 6 represents a generic vertex different from $\{1,2,3,4,5\}$, and the bound was obtained through Lemma 3.8.

The number of occurrences and all bounds provided are up to combinatorial constants, which are independent of $n$ and $p$.

\begin{tabular}{|c|c|c|c|c|}
\hline Pattern & $\begin{array}{l}\text { occur- } \\
\text { rences }\end{array}$ & $\begin{array}{l}\text { bound for } \\
\text { large } p\end{array}$ & $\begin{array}{l}\text { bound for } \\
\text { small } p\end{array}$ & $\begin{array}{c}\text { Lemma } \\
\text { used }\end{array}$ \\
\hline & 1 & $n^{2}(1-p)$ & $p^{3}+n p^{5}+n^{2} p^{7}$ & Lem. 3.8 \\
\hline & $n$ & $n^{2}(1-p)$ & $p^{5}+n p^{7}+n^{2} p^{9}$ & Lem. 3.8 \\
\hline & $n$ & $n^{2}(1-p)$ & $p^{5}+n p^{7}+n^{2} p^{9}$ & Lem. 3.8 \\
\hline & $n$ & $n^{2}(1-p)$ & $\begin{array}{l}p^{6}+n p^{8}+n^{2} p^{10} \\
\leqslant p^{5}+n p^{7}+n^{2} p^{9}\end{array}$ & Lem. 3.8 \\
\hline & $n^{2}$ & $n^{2}(1-p)$ & $p^{7}+n p^{9}+n^{2} p^{11}$ & Lem. 3.8 \\
\hline 1 & $n^{2}$ & $n^{2}(1-p)$ & $p^{5}+n p^{7}+n^{2} p^{9}$ & Lem. 3.8 \\
\hline
\end{tabular}

Table 1: All possible combinations of triangle counts that can occur when expanding $r_{4,1,1}$ and $r_{4,2,1}$, along with the bounds on the corresponding summands. 
Table 2: All possible combinations of triangle indicators that can occur when expanding $r_{4,1,2}$ and $r_{4,2,2}$, along with the bounds on the corresponding summands.

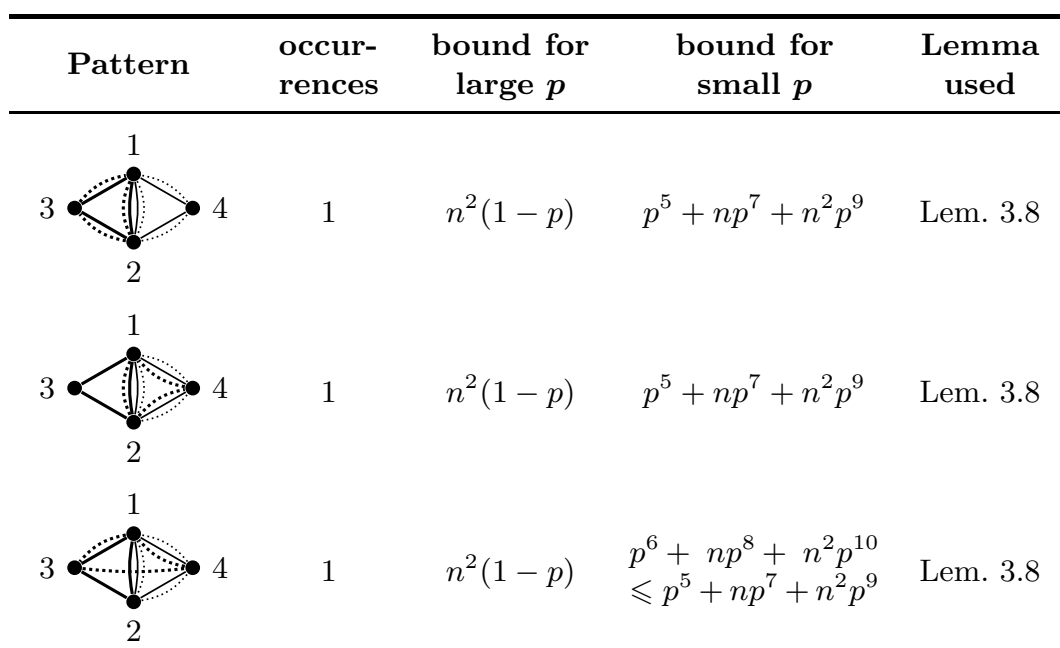

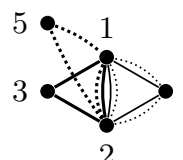

$n \quad n^{2}(1-p) \quad p^{7}+n p^{9}+n^{2} p^{11} \quad$ Lem. 3.8

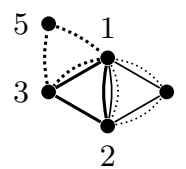

$n \quad n^{2}(1-p) \quad p^{7}+n p^{9}+n^{2} p^{11} \quad$ Lem. 3.8

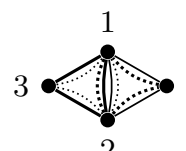

$1 \quad n^{2}(1-p) \quad p^{5}+n p^{7}+n^{2} p^{9} \quad$ Lem. 3.8

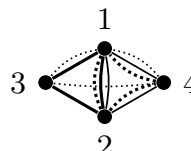

$1 \quad \begin{array}{lll}n^{2}(1-p) & p^{6}+n p^{8}+n^{2} p^{10} \\ \leqslant p^{5}+n p^{7}+n^{2} p^{9} & \text { Lem. 3.8 }\end{array}$

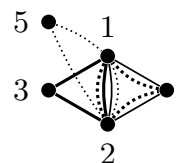

$n \quad n^{2}(1-p) \quad p^{7}+n p^{9}+n^{2} p^{11} \quad$ Lem. 3.8

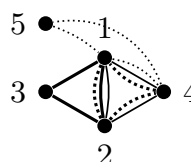

$n \quad n^{2}(1-p) \quad p^{7}+n p^{9}+n^{2} p^{11} \quad$ Lem. 3.8

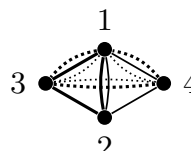

$1 \quad \begin{array}{lll}n^{2}(1-p) & \begin{array}{l}p^{6}+n p^{8}+n^{2} p^{10} \\ \leqslant p^{5}+n p^{7}+n^{2} p^{9}\end{array} \quad \text { Lem. 3.8 }\end{array}$

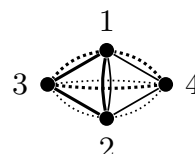

$1 \quad n^{2}(1-p) \quad \begin{array}{ll}p^{6}+n p^{8}+n^{2} p^{10} \\ \leqslant p^{5}+n p^{7}+n^{2} p^{9}\end{array} \quad$ Lem. 3.8

(continued on next page) 
Table 2 (continued from previous page)

\begin{tabular}{ccccc} 
occur- & $\begin{array}{c}\text { bound for } \\
\text { large } \boldsymbol{p}\end{array}$ & $\begin{array}{c}\text { bound for } \\
\text { small } p\end{array}$ & $\begin{array}{c}\text { Lemma } \\
\text { used }\end{array}$ \\
\hline & & $n^{2}(1-p)$ & $\begin{array}{c}p^{8}+n p^{10}+n^{2} p^{12} \\
\leqslant p^{7}+n p^{9}+n^{2} p^{11}\end{array}$ & Lem. 3.8 \\
\hline & & & & \\
\hline
\end{tabular}


Table 3: All possible combinations of triangle indicators that can occur when expanding $r_{4,1,3}$ and $r_{4,2,3}$, along with the bounds on the corresponding summands.

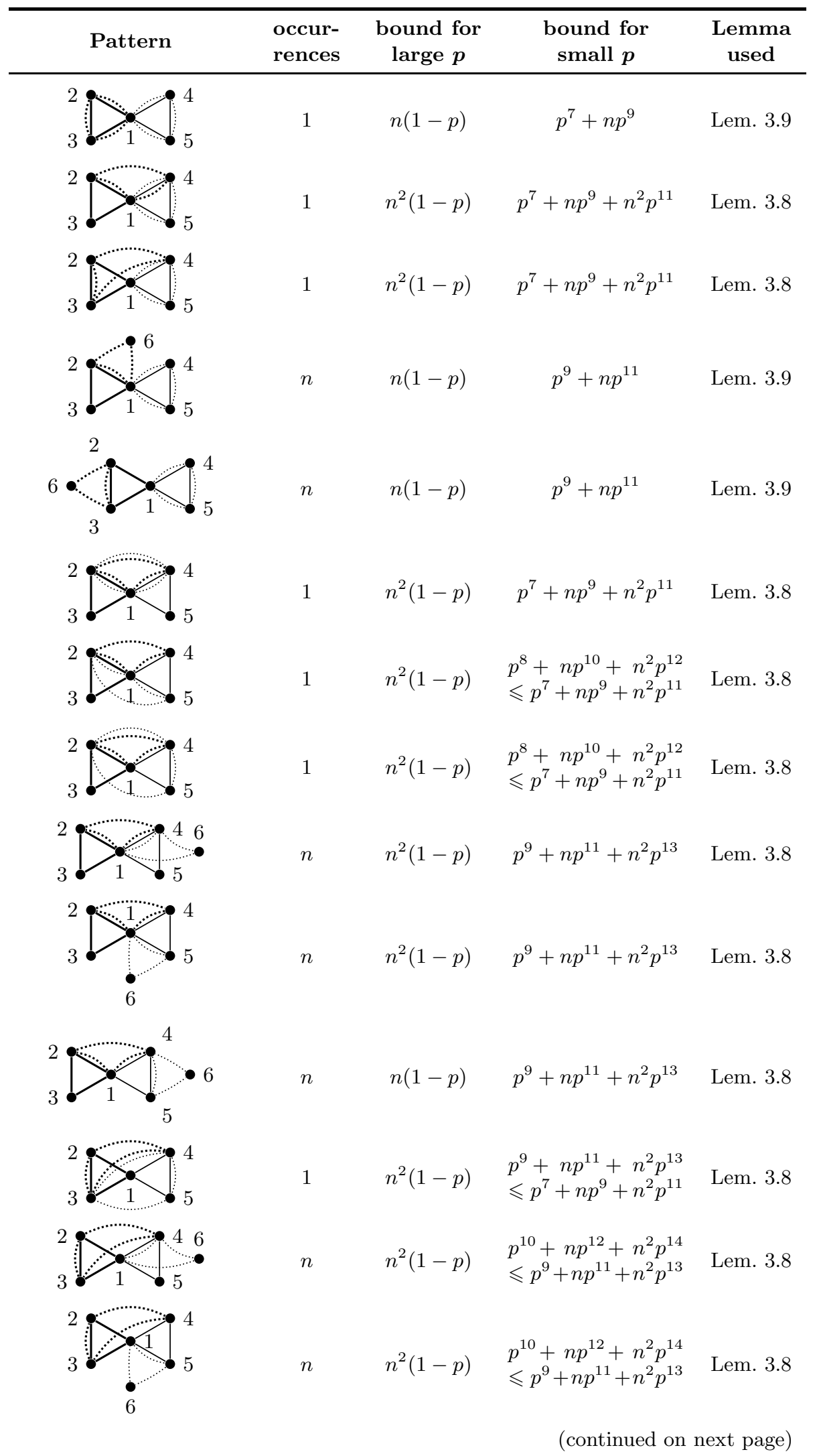


Table 3 (continued from previous page)

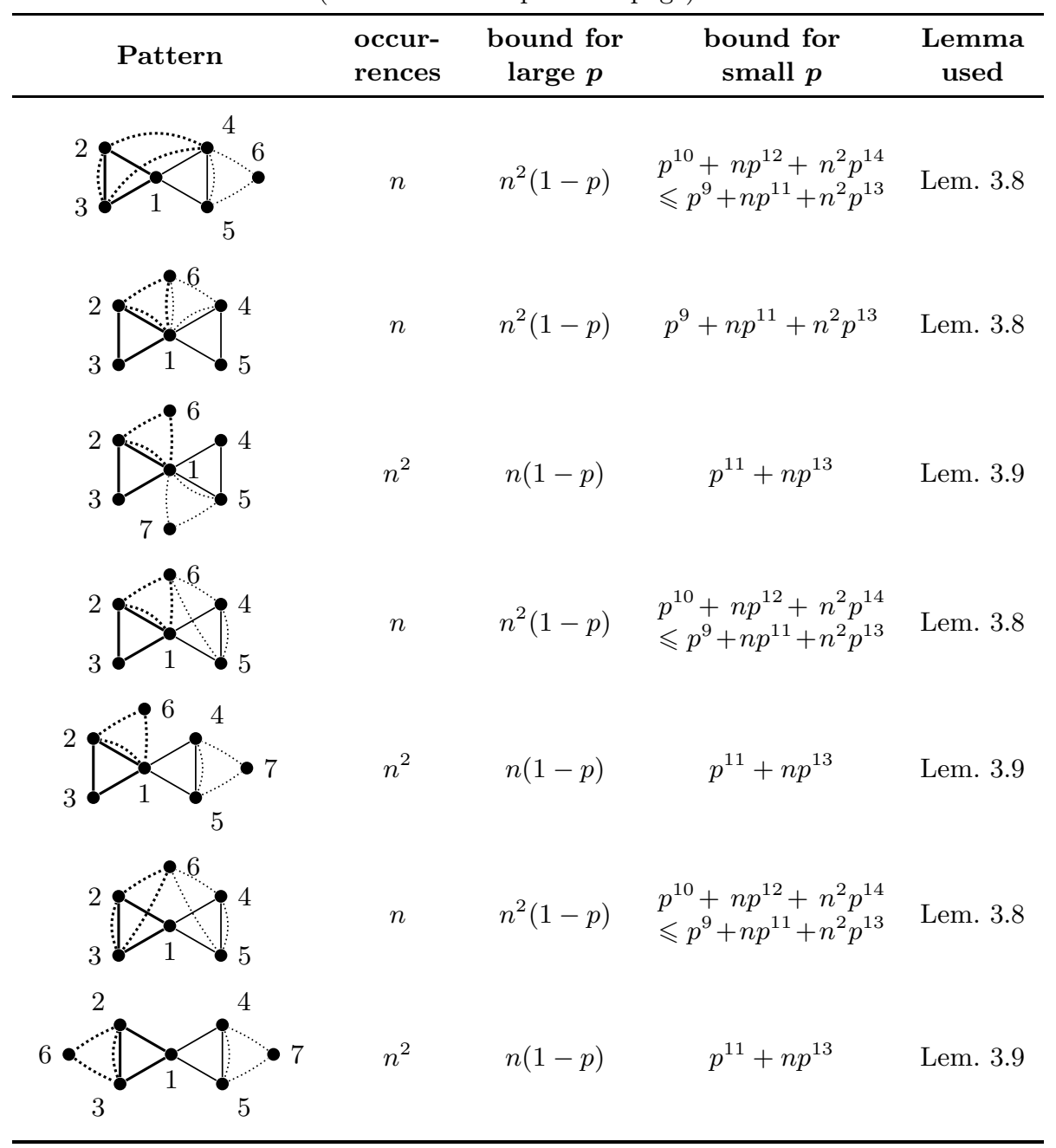

Table 4: All possible combinations of triangle indicators that can occur when expanding $r_{4,1,4}$ and $r_{4,2,4}$, along with the bounds on the corresponding summands.

\begin{tabular}{cccccc}
\hline \multicolumn{1}{c}{ Pattern } & $\begin{array}{c}\text { occur- } \\
\text { rences }\end{array}$ & $\begin{array}{c}\text { bound for } \\
\text { large } \boldsymbol{p}\end{array}$ & $\begin{array}{c}\text { bound for } \\
\text { small } \boldsymbol{p}\end{array}$ & $\begin{array}{c}\text { Lemma } \\
\text { used }\end{array}$ \\
\hline & & & & \\
\end{tabular}


Table 4 (continued from previous page)

\begin{tabular}{ccccc}
\hline & $\begin{array}{c}\text { occur- } \\
\text { rences }\end{array}$ & $\begin{array}{c}\text { bound for } \\
\text { large } \boldsymbol{p}\end{array}$ & $\begin{array}{c}\text { bound for } \\
\text { small } \boldsymbol{p}\end{array}$ & $\begin{array}{c}\text { Lemma } \\
\text { used }\end{array}$ \\
\hline & 1 & $n^{2}(1-p)$ & $p^{9}+n p^{11}+n^{2} p^{13}$ & Lem. [3.8 \\
\hline
\end{tabular}

\section{ACKNOWLEDGMENTS}

I thank Alexander Tikhomirov for very helpful discussions. Thanks go to the Department of Mathematics at the University of Zürich, where part of this article was written, and in particular to Valentin Féray for fruitful discussions and for calling the work of Krokowski. Reichenbachs and Thäle (2017) to my attention. I also thank the Institute of Mathematical Sciences, NUS, for supporting the workshop Workshop on New Directions in Stein's Method in March 2015. This work was supported in parts by NUS Research Grand R-155-000-167-112.

\section{REFERENCES}

A. D. Barbour, M. Karoński and A. Ruciński (1989). A central limit theorem for decomposable random variables with applications to random graphs. J. Combin. Theory Ser. B 47, 125-145.

A. V. Bulinskii (1996). Rate of convergence in the central limit theorem for fields of associated random variables. Theory of Probability and its Applications 40, 136-144. 
L. H. Y. Chen and A. Röllin (2010). Stein couplings for normal approximation. Available at arxiv.org/abs/1003.6039

J. D. Esary, F. Proschan and D. W. Walkup (1967). Association of random variables, with applications. The Annals of Mathematical Statistics 38, 1466-1474.

I. A. Ibragimov and Y. V. Linnik (1971). Independent and stationary sequences of random variables. Wolters-Noordhoff Publishing Groningen.

K. Krokowski, A. Reichenbachs and C. Thäle (2017). Discrete Malliavin-Stein method: BerryEsseen bounds for random graphs and percolation. Ann. Probab. 45, 1071-1109.

C. M. Newman (1980). Normal fluctuations and the FKG inequalities. Comm. Math. Phys. 74, $119-128$.

A. Röllin and N. Ross (2015). Local limit theorems via Landau-Kolmogorov inequalities. Bernoulli 21, 851-880.

N. Ross (2011). Fundamentals of Stein's method. Probab. Surv. 8, 210-293.

A. Ruciński (1988). When are small subgraphs of a random graph normally distributed? Probab. Theory Related Fields. 78, 1-10.

C. Stein (1972). A bound for the error in the normal approximation to the distribution of a sum of dependent random variables. In Proceedings of the Sixth Berkeley Symposium on Mathematical Statistics and Probability (Univ. California, Berkeley, Calif., 1970/1971), Vol. II: Probability theory, pages 583-602, Berkeley, Calif. Univ. California Press.

A. N. Tikhomirov (1980). Convergence rate in the central limit theorem for weakly dependent random variables. Theory Probab. Appl. 25, 790-809. 